\title{
FLOER HOMOLOGY AND SINGULAR KNOTS
}

\author{
PETER OZSVÁTH, ANDRÁS STIPSICZ, AND ZOLTÁN SZABÓ
}

\begin{abstract}
In this paper we define and investigate variants of the link Floer homology introduced by the first and third authors. More precisely, we define Floer homology theories for oriented, singular knots in $S^{3}$ and show that one of these theories can be calculated combinatorially for planar singular knots.
\end{abstract}

\section{INTRODUCTION}

Singular knots arise naturally in the skein theory of ordinary knots, see for example [4]. Moreover, they play a crucial role in the Khovanov-Rozansky categorification of the HOMFLY-PT polynomial [3]. In this paper we define and investigate Floer homology theories for oriented, singular knots in $S^{3}$, whose Euler characteristic is the Alexander polynomial of the knot. The definitions of these theories are generalizations of knot and link Floer homology, [7], [11], [10].

Informally, one can think of a singular knot as an ordinary knot with a finite set of double points. However, for the purpose of skein theory, it is important to endow these objects with some extra structure. To this end an abstract singular knot is a connected, trivalent graph with a distinguished set of edges, called thick edges (and the remaining edges are called thin edges), which satisfies an additional hypothesis: at each vertex, we require two of the edges to be thin, and the third to be thick. An oriented abstract singular knot is obtained by orienting all the edges in such a manner that at each vertex, both thin edges are oriented the same way, while the thick edge is oriented oppositely, see Figure 1(a). For an example of an oriented singular knot see Figure 1(b). Of course, not all abstract singular knots can be oriented in this way; an example for a nonorientable abstract singular knot is shown in Figure 1(c). A singular knot, then, is a PL embedding of an oriented abstract singular knot in $S^{3}$. By contracting all the thick edges to points, we obtain an oriented knot with a finite set of double points.

As it is customary in knot theory, we will mainly study singular knots through their generic projections. In fact, we consider projections which have crossings only among thin edges. Singular knots arise naturally from projections of oriented knots as follows. Given a projection of an ordinary knot, we replace some of its crossings by inserting a

PSO was supported by NSF grant number DMS 0234311.

AS was supported by OTKA T49449.

ZSz was supported by NSF grant number DMS 0107792.

AS and ZSz were partially supported by the EU Marie Curie TOK program BudAlgGeo. 


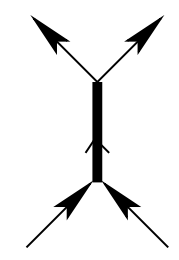

(a)

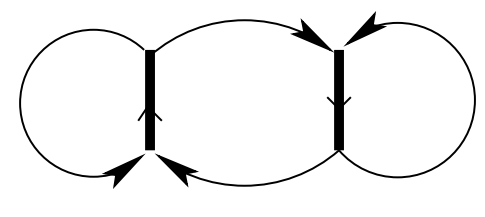

(b)

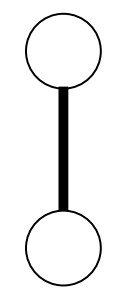

(c)

Figure 1. Singular knots. (a) shows a thick edge, (b) illustrates the orientation convention, while (c) is an example of a singular knot which cannot be oriented.

thick edge, in such a manner that the orientations of the thin edges are preserved. This is illustrated in Figure 2.
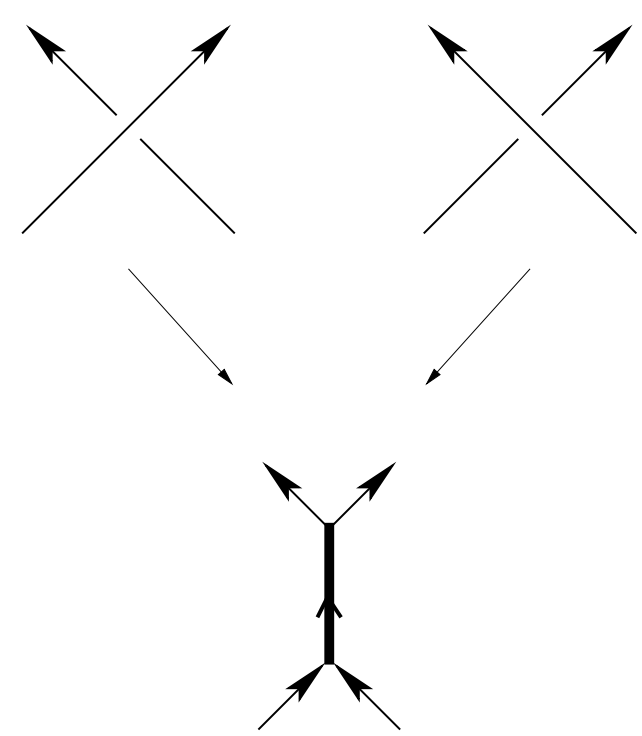

Figure 2. Singularizing knot projections. The diagram shows how to replace a positive/negative crossing by a thick edge in the projection. 
Let $K$ be an oriented, singular knot. The Alexander polynomial $\Delta_{K}(T)$ of $K$ can be defined using the extension of Alexander polynomials of ordinary links via skein theory, as it is discussed in Section 3.

In this paper we construct a bigraded homology theory associated to $K$ whose graded Euler characteristic is determined by $\Delta_{K}(T)$. In fact, there are two variants of this homology theory, $\widetilde{\operatorname{HFS}}(K)$ and $\operatorname{HFS}(K)$, and each splits as

$$
\widetilde{\operatorname{HFS}}(K)=\bigoplus_{s, d} \widetilde{\operatorname{HFS}_{d}}(K, s) \text { and } \operatorname{HFS}(K)=\bigoplus_{s, d} \operatorname{HFS}_{d}(K, s)
$$

where $d$ (corresponding to the homological, or Maslov grading) is an integer, and $s$ (the Alexander grading) is either an integer or a half-integer, depending on the number of thick edges of the singular knot. The group $\operatorname{HFS}(K)$ is a module over the polynomial ring $\mathbb{F}\left[U_{1}, \ldots, U_{\ell}\right]$, where $\ell+1$ denotes the number of thick edges of $K(\ell \geq 0)$ and $\mathbb{F}=\mathbb{Z} / 2 \mathbb{Z}$. In fact, $\operatorname{HFS}(K)$ is gotten as the homology of a chain complex $\operatorname{CFS}(K)$ over this ring. The group $\widetilde{\operatorname{HFS}}(K)$ is obtained by taking the homology of the complex $\widetilde{\mathrm{CFS}}(K)$ gotten by specializing all $U_{i}=0$ in $\operatorname{CFS}(K)$. Multiplication by $U_{i}$ drops Alexander grading by one, and it drops Maslov grading by two.

Note that in the definition of $\operatorname{HFS}(K)$, the number of variables is one less than the number of thick edges; our construction treats one of these edges specially. Thus, these Floer homology groups depend on this choice of a distinguished thick edge on the singular knot; i.e. the resulting group is an invariant of the singular knot equipped with a thick edge. The groups $\widehat{\operatorname{HFS}}(K)$ use all thick edges more symmetrically, and as such depends only on the singular link. See Theorem 2.4 for the invariance statement.

The relationship between these invariants and the Alexander polynomial is spelled out in the following:

Theorem 1.1. For the Floer homology theory $\widetilde{\mathrm{HFS}}$ we have

$$
\sum_{s} \chi\left(\widetilde{\operatorname{HFS}}_{*}(K, s)\right) \cdot T^{s}=(1-T)^{\ell} \cdot \Delta_{K}(T),
$$

where $\ell+1$ denotes the number of thick edges in $K$. For HFS we have

$$
\sum_{s} \chi\left(\operatorname{HFS}_{*}(K, s)\right) \cdot T^{s}=\Delta_{K}(T) .
$$

A singular knot projection gives rise to a natural Heegaard diagram. Using such a diagram, and generalizing the notion of Kauffman states to singular knots (see Section 4 for details), we get a natural interpretation of a set of generators for the Floer homology groups in terms of these extended Kauffman states, similarly to [6, Theorem 1.2].

Theorem 1.2. Let $K$ be a singular knot, and fix a decorated projection $P$ of $K$. Consider the vector space $C(K)$ over $\mathbb{F}$ generated by all generalized Kauffman states of $P$. There is a differential on $C(K)$ whose homology calculates $\operatorname{HFS}(K)$. 
A more precise statement is given in Section 4, where we also give an explicit formula for the bigrading associated to each generalized Kauffman state.

We turn our attention to the Floer homologies of singular knots in the case where the singular knot $K$ is planar, that is, $K$ admits an injective projection to the plane.

Theorem 1.3. Suppose that $K$ is a planar singular knot. Then the group $\operatorname{HFS}(K)$ is determined by the Alexander polynomial $\Delta_{K}(T)$; indeed, the homology $\operatorname{HFS}_{d}(K, s)$ is supported on the line $2 s=d$.

It is more challenging to calculate $\widetilde{\operatorname{HFS}}(K)$. In particular, we exhibit an example which shows that $\widetilde{\operatorname{HFS}}(K, s)$ can be non-trivial even when its Euler characteristic vanishes.

The present paper is organized as follows. In Section 2 we give the definition of $\widetilde{\mathrm{HFS}}$ and HFS. In Section 3 we briefly discuss Alexander polynomials of singular knots. In Section 4 we give the state sum model for the Floer homology theory HFS of singular knots, leading us to the proofs of the theorems announced above. In Section 5 we show an alternative Heegaard diagram for planar singular knots and do some calculations in some special cases.

Most of the material described in this paper was discovered in 2004, while the second author was visiting the Institute for Advanced Study. The invariants for singular knots described here (and some suitable modification of them) form the basis for a cube of resolutions description of knot Floer homology, described in [5], which gives a construction of knot Floer homology quite similar in character to the Khovanov-Rozansky categorification of the HOMFLY-PT polynomial [3]. A different construction of knot Floer homology for singular links has appeared very recently in [1], cf. also [13].

1.1. Acknowledgements. The authors wish to thank the referee for a careful reading and extensive suggestions.

\section{Definition of the Floer homology groups}

2.1. Heegaard diagrams. Consider an oriented surface $\Sigma$ of genus $g$, endowed with two $(g+\ell)$-tuples of mutually pairwise disjoint, embedded circles $\boldsymbol{\alpha}=\alpha_{1}, \ldots, \alpha_{g+\ell}$ and $\boldsymbol{\beta}=\beta_{1}, \ldots, \beta_{g+\ell}$ (where $\ell$ is a non-negative integer), chosen so that $\alpha_{1}, \ldots, \alpha_{g+\ell}$ and $\beta_{1}, \ldots, \beta_{g+\ell}$ determine handlebodies $U_{\alpha}$ and $U_{\beta}$ with $\partial U_{\alpha}=-\partial U_{\beta}=\Sigma$. If $U_{\alpha} \cup_{\Sigma} U_{\beta} \cong$ $S^{3}$, we call $(\Sigma, \boldsymbol{\alpha}, \boldsymbol{\beta})$ a balanced Heegaard diagram for $S^{3}$. A self-indexing Morse function on $S^{3}$ with $\ell+1$ maxima and minima determines a balanced Heegaard diagram for $S^{3}$.

A point $p \in \Sigma-\alpha_{1}-\ldots-\alpha_{g+\ell}-\beta_{1}-\ldots-\beta_{g+\ell}$ determines a gradient flow which connects some index zero and index three critical point. Indeed, given a collection of points $\mathbf{w}=w_{1}, \ldots, w_{a}$ and $\mathbf{z}=z_{1}, \ldots, z_{b}$, each in $\Sigma-\alpha_{1}-\ldots-\alpha_{g+\ell}-\beta_{1}-\ldots-\beta_{g+\ell}$, we obtain an oriented graph $\Gamma_{\mathbf{w}, \mathbf{z}}$, which is oriented compatibly with the flow along the edges corresponding to $w_{i}$, and oppositely for the edges corresponding to $z_{j}$. We suppose moreover that $\Gamma_{\mathbf{w}, \mathbf{z}}$ is a connected graph with vertices of degree three. This 
condition implies that each component $\left\{B_{i}\right\}_{i=1}^{\ell+1}$ of $\Sigma-\beta_{1}-\ldots-\beta_{g+\ell}$ has two points of type $\mathbf{z}$ (denoted by $z_{i}^{1}$ and $z_{i}^{2}$ ), and one point of type $\mathbf{w}$ (which will be denoted by $w_{i}$ ). A similar statement holds for the components $\left\{A_{j}\right\}_{j=1}^{\ell+1}$ of $\Sigma-\alpha_{1}-\ldots-\alpha_{g+\ell}$. In this way we obtain an oriented singular knot in $S^{3}$ by declaring the edges passing through $w_{1}, \ldots, w_{\ell+1}$ to be thick edges. Conversely, it is not hard to see that any singular knot $K \subset S^{3}$ arises in this way, that is, for any $K$ there is a balanced Heegaard diagram compatible with it. An example of such a Heegaard diagram will be given in the next subsection.

2.2. Decorated projections and Heegaard diagrams. Fix a generic projection $P$ of an oriented singular knot $K$. We also fix a generic initial point $Q$ on the projection. We call this data a decorated knot projection for $K$. Given this data, we describe an associated Heegaard diagram, generalizing the one given in [6]. (Note that we have switched here the roles of the $\alpha$ and $\beta$ circles from the conventions from [6].) The handlebody $U_{\alpha}$ is a regular neighborhood of the projection of $K$, and $U_{\beta}$ is its complement in $S^{3}$. Let $X$ and $Y$ be the regions in the projection which contain the distinguished point $Q$. For each region in the complement of the projection other than $X$, we choose a corresponding $\beta$-circle given by the contour of the region. For each crossing in the projection we choose a circle $\alpha_{j}$ supported in a neighborhood of the crossing as it is shown in Figure 3. For each thick edge, we choose a pair of $\alpha$-circles $\alpha_{j}$ and $\alpha_{j+1}$ which are meridians for the two incoming arcs, and also an additional circle $\beta_{i}$, which meets only $\alpha_{j}$ and $\alpha_{j+1}$ in two points apiece. We call $\beta_{i}$ an internal $\beta$-circle for the thick edge. This is illustrated in Figure 4. The diagram also shows how to place the base points $w_{i}$ and $z_{i}^{1}, z_{i}^{2}$ near the thick edge. To complete the construction, finally we omit one of the internal $\beta$-circles. We will take the convention that $w_{\ell+1}$ is the distinguished thick
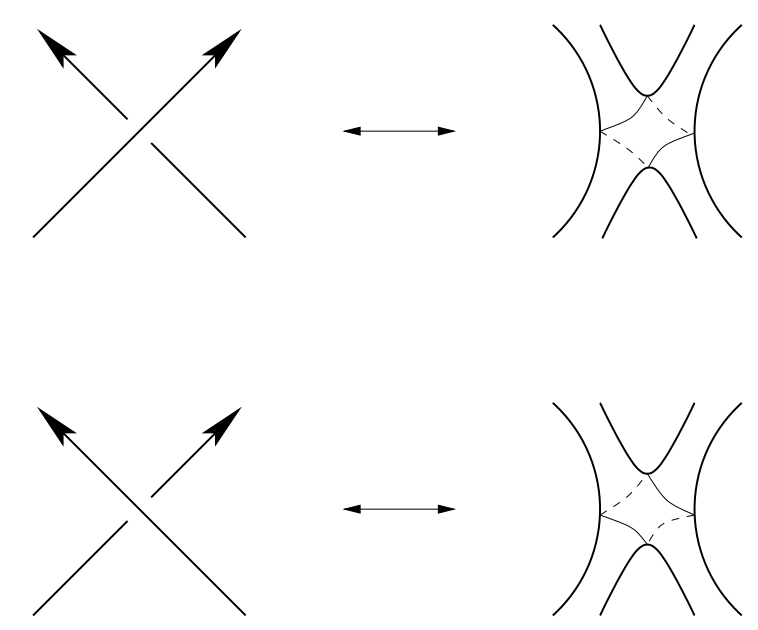

Figure 3. Heegaard diagram at a crossing. The position of the $\alpha$ circle (depending on the sign of the crossing) is shown. 
edge. Moreover, in Section 4, we will find it useful to choose a diagram where the initial point $Q$ lies on a thin edge which points into the distinguished thick edge, and that the internal $\beta$-circle for this thick edge is omitted. It is easy to see that the resulting balanced Heegaard diagram is compatible with the given singular knot.

2.3. Definition of $\widetilde{\operatorname{HFS}}(K)$ and $\operatorname{HFS}(K)$. Suppose now that $K \subset S^{3}$ is a singular knot and $(\Sigma, \boldsymbol{\alpha}, \boldsymbol{\beta}, \mathbf{w}, \mathbf{z})$ is a balanced Heegaard diagram compatible with $K$. As usual (compare [8], [10]), we consider the $(g+\ell)$-fold symmetric power $\operatorname{Sym}^{g+\ell}(\Sigma)$ of the genus- $g$ surface $\Sigma$, equipped with the tori

$$
\mathbb{T}_{\alpha}=\alpha_{1} \times \ldots \times \alpha_{g+\ell} \text { and } \mathbb{T}_{\beta}=\beta_{1} \times \ldots \times \beta_{g+\ell}
$$

in $\operatorname{Sym}^{g+\ell}(\Sigma)$. Let $\pi_{2}(\mathbf{x}, \mathbf{y})$ denote the set of homology classes of topological disks connecting the intersection points $\mathbf{x}, \mathbf{y} \in \mathbb{T}_{\alpha} \cap \mathbb{T}_{\beta}$. For $p \in \Sigma-\alpha_{1}-\ldots-\alpha_{g+\ell}-$ $\beta_{1}-\ldots-\beta_{g+\ell}$ and $\phi \in \pi_{2}(\mathbf{x}, \mathbf{y})$ we define $n_{p}(\phi)$ as the intersection of $\phi$ with $V_{p}=$ $\{p\} \times \operatorname{Sym}^{g+\ell-1}(\Sigma)$. We decompose the complement of the $\alpha_{i}$ and $\beta_{j}$ into its components

$$
\Sigma-\alpha_{1}-\ldots-\alpha_{g+\ell}-\beta_{1}-\ldots-\beta_{g+\ell}=\coprod_{k} C_{k} .
$$

Given $\phi \in \pi_{2}(\mathbf{x}, \mathbf{y})$, the domain $\mathcal{D}(\phi)$ associated to $\phi$ is the formal linear combination $\mathcal{D}(\phi)=\sum_{j} n_{j} C_{j}$, where here $n_{j}=n_{c_{j}}(\phi)$ for any choice of $c_{j} \in C_{j}$. The homology class of $\phi$ is uniquely determined by its associated domain. We say that the domain $\mathcal{D}$ is nonnegative (writing $\mathcal{D} \geq 0$ ) if $\mathcal{D}=\sum_{j} n_{j} C_{j}$ with $n_{j} \geq 0$ for all $j$.

Two functions

$$
A^{\prime}:\left(\mathbb{T}_{\alpha} \cap \mathbb{T}_{\beta}\right) \times\left(\mathbb{T}_{\alpha} \cap \mathbb{T}_{\beta}\right) \longrightarrow \mathbb{Z}
$$

and

$$
N^{\prime}:\left(\mathbb{T}_{\alpha} \cap \mathbb{T}_{\beta}\right) \times\left(\mathbb{T}_{\alpha} \cap \mathbb{T}_{\beta}\right) \longrightarrow \mathbb{Z}
$$
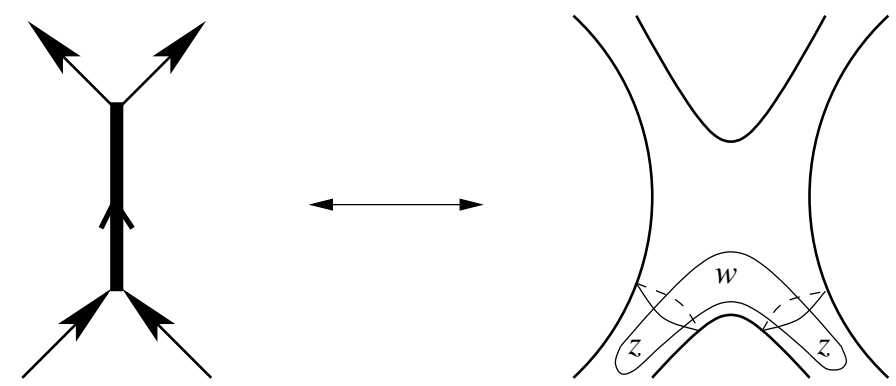

Figure 4. Heegaard diagram at a thick edge. The diagram describes the $\alpha$-cirles and the internal $\beta$-circle corresponding to a thick edge, together with the base points $w$ and two $z$ 's. 
can be defined by the formulas

$$
A^{\prime}(\mathbf{x}, \mathbf{y})=\sum_{i=1}^{\ell+1}\left(2 n_{w_{i}}(\phi)-n_{z_{i}^{1}}(\phi)-n_{z_{i}^{2}}(\phi)\right)
$$

and

$$
N^{\prime}(\mathbf{x}, \mathbf{y})=\mu(\phi)-2 \sum_{i=1}^{\ell+1} n_{w_{i}}(\phi)
$$

where $\phi \in \pi_{2}(\mathbf{x}, \mathbf{y})$ is arbitrary, and $\mu(\phi)$ is the Maslov index of $\phi \in \pi_{2}(\mathbf{x}, \mathbf{y})$.

Lemma 2.1. The functions $A^{\prime}$ and $N^{\prime}$ are well-defined, i.e. they are independent of the choice of $\phi \in \pi_{2}(\mathbf{x}, \mathbf{y})$. Moreover, given $\mathbf{x}, \mathbf{y}, \mathbf{w} \in \mathbb{T}_{\alpha} \cap \mathbb{T}_{\beta}$, we have that $A^{\prime}(\mathbf{x}, \mathbf{y})+$ $A^{\prime}(\mathbf{y}, \mathbf{w})=A^{\prime}(\mathbf{x}, \mathbf{w})$ and $N^{\prime}(\mathbf{x}, \mathbf{y})+N^{\prime}(\mathbf{y}, \mathbf{w})=N^{\prime}(\mathbf{x}, \mathbf{w})$.

Proof. Consider $\phi$ and $\phi^{\prime} \in \pi_{2}(\mathbf{x}, \mathbf{y})$. The difference $D=\mathcal{D}(\phi)-\mathcal{D}\left(\phi^{\prime}\right)$ of their domains specifies a two-chain whose boundary is a sum of $\alpha$ - and $\beta$-circles. Since $H_{1}(\Sigma)$ is spanned by the images of the two $g$-dimensional subspaces spanned by the $\left\{\left[\alpha_{i}\right]\right\}_{i=1}^{g+\ell}$ and $\left\{\left[\beta_{i}\right]\right\}_{i=1}^{g+\ell}$, it follows that any such domain can be written as a sum of some $D_{1}$ and $D_{2}$, where

$$
D_{1}=\sum_{i=1}^{\ell+1} a_{i} \cdot A_{i} \quad \text { and } \quad D_{2}=\sum_{i=1}^{\ell+1} b_{i} \cdot B_{i},
$$

where $\left\{A_{i}\right\}_{i=1}^{\ell+1}$ resp. $\left\{B_{i}\right\}_{i=1}^{\ell+1}$ are the components of $\Sigma-\alpha_{1}-\ldots-\alpha_{g+\ell}$ resp. $\Sigma-\beta_{1}-$ $\ldots-\beta_{g+\ell}$, and $a_{i}, b_{i} \in \mathbb{Z}$. Now, our combinatorial condition on the Heegaard diagram ensures that each component $A_{i}$ or $B_{j}$ contains two points of $\mathbf{z}$ and one point of $\mathbf{w}$ (corresponding to a thick edge). Since these latter points contribute to the sum in $A^{\prime}$ with multiplicity two, it follows readily that

$$
\begin{aligned}
& \sum_{i=1}^{\ell+1}\left(2 n_{w_{i}}(\phi)-n_{z_{i}^{1}}(\phi)-n_{z_{i}^{2}}(\phi)\right) \\
& =\sum_{i=1}^{\ell+1}\left(2 n_{w_{i}}\left(\phi^{\prime}\right)-n_{z_{i}^{1}}\left(\phi^{\prime}\right)-n_{z_{i}^{2}}\left(\phi^{\prime}\right)\right) ;
\end{aligned}
$$

i.e. $A^{\prime}$ is well-defined. Well-definedness of $N^{\prime}$ follows from standard properties of the Maslov index, see [10, Proposition 4.1]. Additivity of these quantities is straightforward.

It follows from Lemma 2.1 that there are functions $A: \mathbb{T}_{\alpha} \cap \mathbb{T}_{\beta} \longrightarrow \mathbb{Z}$ and $N: \mathbb{T}_{\alpha} \cap$ $\mathbb{T}_{\beta} \longrightarrow \mathbb{Z}$, uniquely characterized up to an overall translation by the property that $A(\mathbf{x})-A(\mathbf{y})=A^{\prime}(\mathbf{x}, \mathbf{y})$ and $N(\mathbf{x})-N(\mathbf{y})=N^{\prime}(\mathbf{x}, \mathbf{y})$. It will be useful to have the following 
Lemma 2.2. Given $\mathbf{x} \in \mathbb{T}_{\alpha} \cap \mathbb{T}_{\beta}$, there is a unique $\phi \in \pi_{2}(\mathbf{x}, \mathbf{x})$ with $n_{\mathbf{w}}(\phi)=n_{\mathbf{z}}(\phi)=0$.

Proof. Notice first that the trivial class $\phi_{0} \in \pi_{2}(\mathbf{x}, \mathbf{x})$ has the required property. Any other class in $\pi_{2}(\mathbf{x}, \mathbf{x})$ differs from $\phi_{0}$ by adding $D_{1}$ and $D_{2}$ as above. Now the condition on $n_{\mathbf{w}}$ and $n_{\mathbf{z}}$ implies that for any $a_{i}$ in $D_{1}$ and $b_{j}$ in $D_{2}$ with the property that $A_{i} \cap B_{j}$ contains some $z_{i}$ or $w_{i}$ we have that $b_{j}=-a_{i}$. Using this repeatedly, together with the fact that $\Gamma_{\mathbf{w}, \mathbf{z}}$ is connected, we see that $D_{1}+D_{2}$ is zero, concluding the proof.

We define the complex $\operatorname{CFS}(K)$ to be the chain complex freely generated over $\mathbb{F}\left[U_{1}, \ldots, U_{\ell}\right]$ (with $\mathbb{F}=\mathbb{Z} / 2 \mathbb{Z}$ ) by the intersection points $\mathbb{T}_{\alpha} \cap \mathbb{T}_{\beta}$, endowed with the differential

$$
\partial(\mathbf{x})=\sum_{\mathbf{y} \in \mathbb{T}_{\alpha} \cap \mathbb{T}_{\beta}} \sum_{\left\{\phi \in \pi_{2}(\mathbf{x}, \mathbf{y}) \mid \mu(\phi)=1, n_{\mathbf{w}}(\phi)=0\right\}} \# \widehat{\mathcal{M}}(\phi) \cdot\left(\prod_{i=1}^{\ell} U_{i}^{\left(n_{z_{i}^{1}}(\phi)+n_{z_{i}^{2}}(\phi)\right)}\right) \mathbf{y}
$$

where $\# \widehat{\mathcal{M}}(\phi)$ is the mod 2 count of holomorphic representatives of $\phi \in \pi_{2}(\mathbf{x}, \mathbf{y})$ up to reparametrization.

The functions $A$ and $N$ defined above induce gradings on $\operatorname{CFS}(K)$, the Alexander and algebraic gradings, with the convention that multiplication by $U_{i}$ drops $A$-grading by one, and preserves $N$.

For the case of non-singular knots, knot Floer homology is bigraded, with Alexander and Maslov degrees. The Maslov degree of [7] behaves differently from the grading $N$ introduced here: multiplication by $U_{i}$ drops Maslov grading, while it preserves $N$. In fact, we could define the following Maslov grading for the case of singular links by taking

$$
M^{\prime}(\mathbf{x}, \mathbf{y})=\mu(\phi)+2 \cdot \sum_{i=1}^{\ell+1}\left(n_{w_{i}}(\phi)-n_{z_{i}^{1}}(\phi)-n_{z_{i}^{2}}(\phi)\right) .
$$

This is analogous to the Maslov grading for knots, and in particular, with respect to the induced grading on $\operatorname{CFS}(K)$, multiplication by any $U_{i}$ drops this grading by two. Note that we have the following easily checked relationship between the three gradings:

$$
N^{\prime}=M^{\prime}-2 A^{\prime}
$$

The complex $\widetilde{\mathrm{CFS}}(K)$ is the chain complex we get by setting all $U_{i}=0(i=1, \ldots, \ell)$ in $\operatorname{CFS}(K)$. Equivalently, it is the free Abelian group generated over $\mathbb{F}$ by $\mathbb{T}_{\alpha} \cap \mathbb{T}_{\beta}$, endowed with the differential

$$
\widetilde{\partial}(\mathbf{x})=\sum_{\mathbf{y} \in \mathbb{T}_{\alpha} \cap \mathbb{T}_{\beta}} \sum_{\left\{\phi \in \pi_{2}(\mathbf{x}, \mathbf{y}) \mid \mu(\phi)=1, n_{\mathbf{w}}(\phi)=0, n_{\mathbf{z}}(\phi)=0\right\}} \# \widehat{\mathcal{M}}(\phi) \cdot \mathbf{y} .
$$

Lemma 2.3. The maps $\widetilde{\partial}$ and $\partial$ induce differentials on $\widetilde{\mathrm{CFS}}$ and CFS which drop the $N$-grading by one, and preserve the A-grading. 
Proof. The sum defining $\widetilde{\partial}$ is finite, according to Lemma 2.2. For $\partial$, we argue that for fixed $\mathbf{x}$ and $\mathbf{y}$, there are only finitely many $\phi \in \pi_{2}(\mathbf{x}, \mathbf{y})$ with $n_{\mathbf{w}}(\phi)=0$ and $\mathcal{D}(\phi) \geq 0$. To see this, observe that the homology class of any $\phi$ with $n_{\mathbf{w}}(\phi)=0$ is uniquely determined by $n_{\mathbf{z}}(\phi)$ (by Lemma 2.2), and moreover according to Lemma 2.1, the sum

$$
\sum_{i=1}^{\ell+1} n_{z_{i}^{1}}(\phi)+n_{z_{i}^{2}}(\phi)
$$

is independent of the choice of $\phi$ (since it is $A(\mathbf{y})-A(\mathbf{x}))$.

Since the coefficients in our theories are chosen in $\mathbb{F}=\mathbb{Z} / 2 \mathbb{Z}$, the arguments of [10] apply. Now the proof that $\widetilde{\partial}^{2}=0=\partial^{2}$ is an adaptation of [10, Lemma 4.3].

Note that, we have so far defined $N$ and $A$ only up to an overall additive constant. The indeterminacy in $N$ can be easily removed with the following observation. Suppose that we set all the $U_{i}=1$ in the definition of CFS (equivalently, consider the differential which counts holomorphic disks with $n_{\mathbf{w}}(\phi)=0$, and ignore the reference points $\mathbf{z}$ ). In this way we obtain a chain complex whose homology is (up to an overall degree shift) isomorphic to $H_{*}\left(T^{\ell}\right)$, see [10, Theorem 4.5]. Note that by Lemma 2.2, it follows that this diagram is admissible, so that the theorem applies. We choose $N$ so that the homology group is isomorphic to $H_{*}\left(T^{\ell}\right)$, with a shift in the grading making the top-most homology supported in degree zero.

The indeterminacy of $A$ can be removed in an invariant manner using relative $\operatorname{Spin}^{c}$ structures (compare [14]), though this is a slightly awkward approach for the present applications. Rather, we will leave the $A$-grading well-defined only up to an overall shift, which could be removed with the help of the state sum formulae of Section 4 . We now write

$$
\widetilde{\mathrm{CFS}}(K)=\bigoplus_{d, s} \widetilde{\mathrm{CFS}_{d}}(K, s) \text { and } \operatorname{CFS}(K)=\bigoplus_{d, s} \operatorname{CFS}_{d}(K, s)
$$

where here $\widetilde{\mathrm{CFS}}_{d}(K, s)$ and $\mathrm{CFS}_{d}(K, s)$ are generated by elements with $M=d \in \mathbb{Z}$ and $A=s$. (Presently, the grading by $s$ is well-defined only up to an additive constant.)

Theorem 2.4. Consider the homology groups

$$
\widetilde{\operatorname{HFS}}(K)=\bigoplus_{d, s} \widetilde{\operatorname{HFS}_{d}}(K, s) \text { and } \operatorname{HFS}(K)=\bigoplus_{d, s} \operatorname{HFS}_{d}(K, s)
$$

of the chain complexes $(\widetilde{\mathrm{CFS}}(K), \widetilde{\partial})$ and $(\mathrm{CFS}(K), \partial)$, where here $d \in \mathbb{Z}$ is an absolute integral grading and $s$ (which is in $\mathbb{Z}$ or $\mathbb{Z}+\frac{1}{2}$, depending on the parity of the number of thick edges) is a relative integral grading. Then, $\operatorname{HFS}(K)$ is an invariant of the singular knot $K$, together with the distinguished thick edge; while $\widetilde{\operatorname{HFS}}(K)$ is an invariant of the singular knot. 
Proof. By Morse theory it is known that any two pointed Heegaard diagrams for a fixed singular knot can be connected by a sequence of (pointed) handleslides, isotopies, stabilizations and birth/death of index $0 / 1$ (and 2/3) cancelling handles. Using the proof of [12, Lemma 1.1] we can avoid the appearance of cancelling $0 / 1$ and $2 / 3$ handles. For the remaining moves the proof of the invariance of the knot Floer homology groups follows the same lines as the proof of [10, Theorem 4.7].

2.4. Other constructions. There are other variants of Floer homology for singular knots, which generalize the constructions we describe here. For example, we could introduce a variable $U_{\ell+1}$ for the final thick edge. The corresponding theory is the analogue for singular knots of $\mathrm{HFK}^{-}$for knots (while the construction HFS here plays the role of $\widehat{\mathrm{HFK}})$.

Indeed, this can be further generalized as follows: consider the chain complex $C^{\prime}$ which is freely generated by $\mathbb{T}_{\alpha} \cap \mathbb{T}_{\beta}$ over the ring $\mathbb{F}\left[U_{1}, \ldots, U_{m}\right]$, where $m$ denotes the number of thin edges in our diagram, and endowed with the differential:

$$
\partial^{\prime}(\mathbf{x})=\sum_{\mathbf{y} \in \mathbb{T}_{\alpha} \cap \mathbb{T}_{\beta}} \sum_{\left\{\phi \in \pi_{2}(\mathbf{x}, \mathbf{y})\right.} \# \widehat{\left.\mu(\phi)=1, n_{\mathbf{w}}(\phi)=0\right\}} \underset{\mathcal{M}}{ }(\phi) \cdot\left(\prod_{i=1}^{m} U_{i}^{n_{z_{i}}}(\phi)\right) \mathbf{y} .
$$

The chain complex (CFS, $\partial$ ) described earlier can be thought of as the chain complex over the quotient ring where we set $U_{e}=U_{e^{\prime}}$, if $e$ and $e^{\prime}$ are two edges which point into the same vertex; and also for some edge $e_{0}$, we set $U_{e_{0}}=0$. See [5] for related constructions.

\section{Alexander POlynomial of Singular LinKS}

State sum formulas for the Alexander polynomial of a smooth knot were introduced in [2]. Our aim in this section is to recall the Alexander polynomial for singular links, and describe a state sum description for it, cf. also [4].

Proposition 3.1. There is a unique extension $\widetilde{\Delta}_{K}(T)$ of the one-variable symmetrized Alexander polynomial $\Delta_{K}(T)$ for non-singular, oriented links to singular, oriented links, which is characterized by the skein relations

$$
\begin{aligned}
& \widetilde{\Delta}_{K^{+}}(T)=\widetilde{\Delta}_{K}(T)+T^{\frac{1}{2}} \cdot \widetilde{\Delta}_{K^{o}}(T) \\
& \widetilde{\Delta}_{K^{-}}(T)=\widetilde{\Delta}_{K}(T)+T^{-\frac{1}{2}} \cdot \widetilde{\Delta}_{K^{o}}(T),
\end{aligned}
$$

where here $K^{o}$ denotes the resolution of $K$ at a singular point $v$, and $K^{+}$and $K^{-}$denote the positive and negative resolutions at $v$ (cf. Figure 5). 


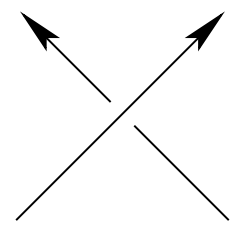

$K^{+}$

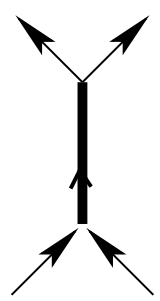

$K$

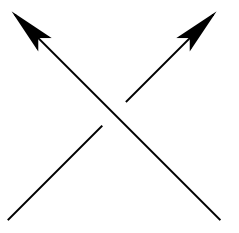

$K^{-}$

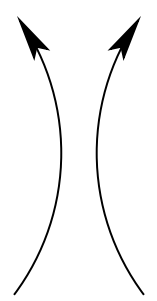

$K^{o}$

Figure 5. Resolutions at a thick edge.

Proof. Uniqueness of such an extension follows from simple induction on the number of singular points, since $K^{ \pm}$and $K^{o}$ have fewer singular points than $K$. The fact that $\widetilde{\Delta}_{K}(T)$ is well-defined follows from a similar inductive argument together with the fact that, because of the ordinary skein relation for the Alexander polynomial, the two equations above provide coherent results.

From now on, the Alexander polynomial $\widetilde{\Delta}_{K}(T)$ for a singular knot $K$ will be denoted simply by $\Delta_{K}(T)$. Now we turn to the state sum description of $\Delta_{K}(T)$ for singular knots. To this end, first we recall the definition of Kauffman states for a singular link projection. Fix a decorated projection $P$ for a singular link $K$ with initial point $Q$ and contract the thick edges to points. Let $\operatorname{Cr}(P)$ and $R(P)$ denote the set of crossings and the set of regions in the complement of the projection, resp. Let $X$ and $Y$ be the two regions which contain the edge containing $Q$. A Kauffman state is a bijection $x: \operatorname{Cr}(P) \longrightarrow R(P)-X-Y$ which assigns to each crossing $v \in \operatorname{Cr}(P)$ one of the (up to) four regions which meet at $v$. Let $x$ be a Kauffman state and $v$ a crossing in the projection. There is a local contribution $Z_{v}(x)$ defined according to which quadrant $x$ assigns to $v$, and the type of $v$ (i.e., whether it is singular, positive, or negative). When $v$ is non-singular, this local contribution takes values $Z_{v}(x) \in\left\{1, \pm T^{ \pm \frac{1}{2}}\right\}$, and when $v$ is singular, $Z_{v}(x) \in\left\{0,1,-T^{\frac{1}{2}}-T^{-\frac{1}{2}}\right\}$. The precise rules are illustrated in Figure 6 . This definition can be regarded as an appropriate extension of the local contributions for Kauffman states of ordinary links. In the case of ordinary links, the appropriate sum of the local contributions of Kauffman states provides the Alexander polynomial; 
Proposition 3.2 generalizes this fact to singular links. We note here that for our later purposes a refinement of Kauffman states (which we will call generalized Kauffman states) will be introduced in Section 4.

Proposition 3.2. Fix a decorated projection $P$ of the oriented link $K$, let $\operatorname{Cr}(P)$ denote the set of crossings in the projection while $X(P)$ denotes its set of Kauffman states. Then the Alexander polynomial $\Delta_{K}(T)$ is calculated using the state sum formula

$$
\Delta_{K}(T)=\sum_{x \in X(P)}\left(\prod_{v \in \operatorname{Cr}(P)} Z_{v}(x)\right)
$$

Proof. The state sum formula for $K^{o}$ can be thought of as given by a state sum formula for states of $K$, where the local contribution at the resolved point contributes as in Figure 7. Since the state sum formula satisfies the skein relation of Proposition 3.1, and the identity of the proposition is known to hold for a non-singular link, the formula of (5) for $\Delta_{K}(T)$ follows at once.
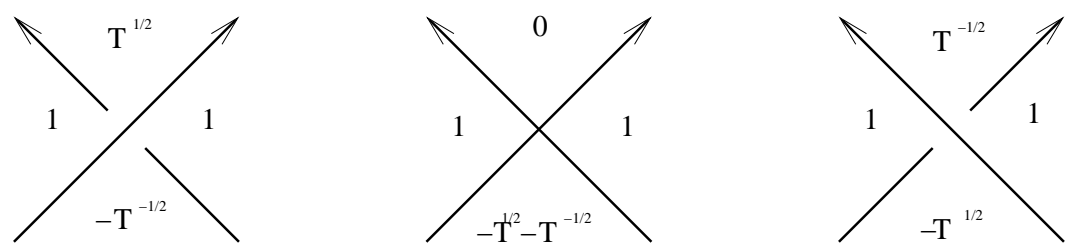

FiguRE 6. Local contributions. We illustrate here the local contribution function $Z_{v}(x)$ of a Kauffman state at a given crossing $v$. The left and right diagrams illustrate the case where the crossing is a non-singular point of $K$, while the middle one illustrates the case where $v$ is a singular point.

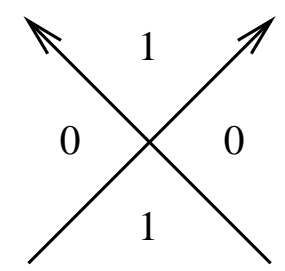

FIGURE 7. Local contributions at a resolved point. 


\section{Kauffman states And the Chain COMPleX}

Our aim is to give a simple set of generators for a chain complex whose homology is isomorphic to $\operatorname{HFS}(K)$. These generators can be described concretely in terms of a suitable generalization of Kauffman states, which we describe presently.

Fix a decorated projection $P$ for a singular knot $K$ with initial point $Q$. We contract all the thick edges, so that they become crossings of the projection. However, the four quadrants around a singular point $v$ do not play equal roles. There are two quadrants, the side quadrants which correspond to regions which used to contain the thick edge in their boundary, which we denote $A_{v}$ and $C_{v}$. There are two remaing quadrants: the top quadrant $B_{v}$, which is pointed towards by the thick edge, and the bottom quadrant $D_{v}$. We define four quadrants $A_{v}, B_{v}, C_{v}$, and $D_{v}$ at the ordinary crossings analogously.

We define a set, the set of Kauffman corners at $v$, for each crossing $v$ of $K$, which depends on whether $v$ is an ordinary crossing or a contracted thick edge. If $v$ is an ordinary crossing, the Kauffman corners are the four corners $A, B, C$, or $D$ of the crossing $v$. If $v$ is a singular crossing, the Kauffman corners correspond to $A, C, D^{+}$, and $D^{-}$, where both $D^{+}$and $D^{-}$belong to the bottom corner $D$.

Let $R(P)$ denote the set of regions in the complement of the projection. Let $X$ and $Y$ be the two regions which contain the edge containing $Q$. Let $\operatorname{Cr}(P)$ denote the set of crossings of $P$ (i.e. $\mathrm{Cr}(P)$ consists of the ordinary crossings and also the contracted thick edges). A generalized Kauffman state for a singular knot is a map $x$ which associates to each crossing $v \in \operatorname{Cr}(P)$ one of its four allowed Kauffman corners, with the constraint that in each allowed region in $R(P)$, there is a unique Kauffman corner in the image of $x$, compare [2]. (Note that this is a very mild generalization of the notion from Section 3: there, we had four states corresponding to four quadrants, except one corner in the singular case contributed $-T^{\frac{1}{2}}-T^{-\frac{1}{2}}$; for our present purposes, it is convenient to think of both terms as corresponding to two different states $D^{+}$and $D^{-}$.)

Each Kauffman corner has a local Maslov grading $M_{v}$, which is zero on all Kauffman corners except for $D$ (and $D^{+}, D^{-}$at a singular point), where it is $\mp 1$ according to the sign of the crossing. This choice of signs is illustrated in Figure 8. The Maslov grading of a generalized Kauffman state is the sum of local Maslov gradings over each crossing of $K$. (Notice that at the singular point no value at $B$ is specified, since when $v$ is a singular point, $B$ is not a Kauffman corner.)

Similarly, each Kauffman corner has a local Alexander grading $S_{v}$, defined as follows. If $v$ is an ordinary positive crossing then $S_{v}(A)=S_{v}(C)=0, S_{v}(B)=1 / 2$ and $S_{v}(D)=$ $-1 / 2$; while if $v$ is negative, then $S_{v}(A)=S_{v}(C)=0, S_{v}(B)=-1 / 2$ and $S_{v}(D)=1 / 2$; finally, if $v$ is a singular crossing, then $S_{v}(A)=S_{v}(C)=0$ and $S_{v}\left(D^{-}\right)=-1 / 2$, $S_{v}\left(D^{+}\right)=1 / 2$, see Figure 9 . The Alexander grading $S(x)$ of a Kauffman state $x$ is given as a sum of local Alexander gradings.

Fix a decorated projection for a singular knot $K$, with the property that the distinguished edge $Q$ is just underneath a singular crossing $p$. Consider next the associated 

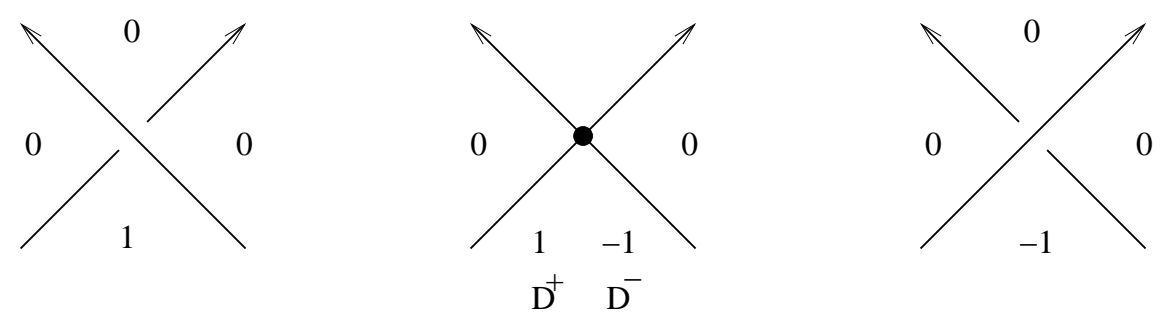

Figure 8. The local Maslov grading $M_{v}$ is illustrated at a crossing $v$.
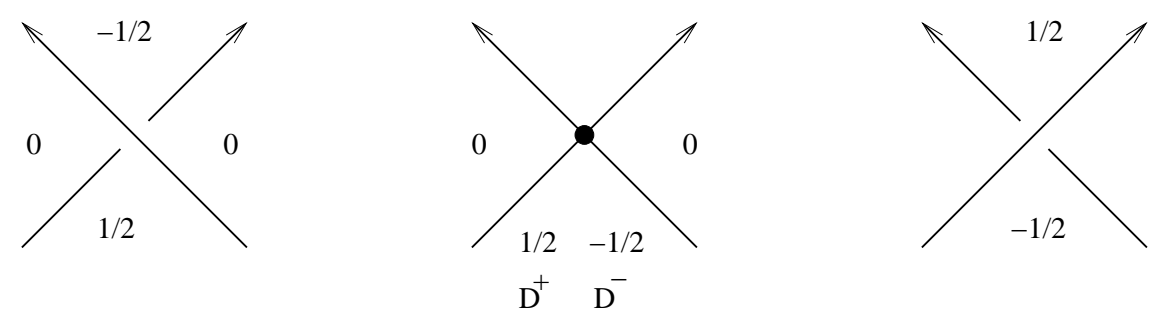

FiguRE $9 . \quad$ The local Alexander grading $S_{v}$ is illustrated at a
crossing $v$.

Heegaard diagram for $K$ described in Section 2.2. Leave out the internal $\beta$-circle $\beta_{p}$ associated to the singular crossing $p$ which lies above $Q$. Moreover, $p$ corresponds to the special thick edge. It is easy to see that there is a Kauffman state associated to each $\mathbf{x} \in \mathbb{T}_{\alpha} \cap \mathbb{T}_{\beta}$, determined as follows. Suppose that $v$ is a singular crossing other than $p$, and let $\alpha_{j}$ and $\alpha_{j+1}$ be the two circles supported in a neighborhood of the singular point $v$. Let $\beta_{v}$ be the corresponding internal $\beta$ circle, and write $\beta_{A}, \beta_{B}, \beta_{C}$, and $\beta_{D}$ be the four $\beta$-circles corresponding to the four quadrants meeting at $v$. Our tuple $\mathbf{x}$ contains exactly one of the four points

$$
\left\{a_{v}, c_{v}, d_{v}^{+}, d_{v}^{-}\right\}=\left(\alpha_{j} \cup \alpha_{j+1}\right) \cap\left(\beta_{A} \cup \beta_{C} \cup \beta_{D}\right),
$$

where $a_{v} \in \beta_{A}, d_{v}^{ \pm} \in \beta_{D}$, and $c_{v} \in \beta_{C}$ (note that the intersection cannot contain more points since $\beta_{j}=\beta_{v}$ meets only $\alpha_{j}$ and $\left.\alpha_{j+1}\right)$. We distinguish $d_{v}^{+}$and $d_{v}^{-}$by the following convention: $d_{v}^{+}$lies on the same $\alpha$-circle as $c_{v}$. Now, our Kauffman state is determined by $x(v)=A_{v}, D_{v}^{+}, D_{v}^{-}$, and $C_{v}$, if $\mathbf{x}$ contains $a_{v}, d_{v}^{+}, d_{v}^{-}$, and $c_{v}$ respectively. The local picture at such a generic singular crossing is illustrated in Figure 10. At the singular point $p$, a similar but somewhat simpler picture applies (the internal $\beta$-circle is missing, as is one of the $\beta$-arcs corresponding to a distinguished edge). The Kauffman state at an ordinary crossing is determined similarly, cf. [6]. 

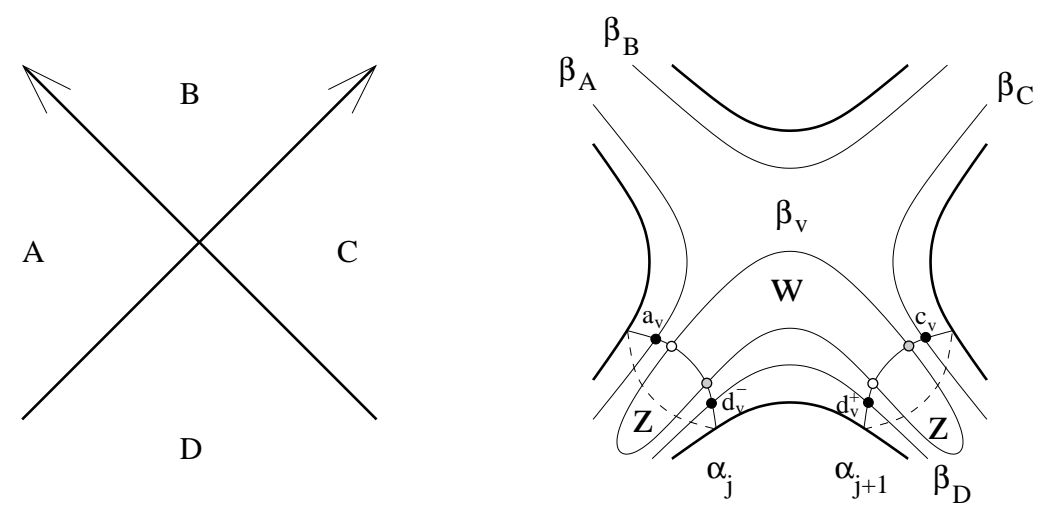

Figure 10. The local picture of the Heegaard diagram near a singular point $v$. There are four unlabelled intersection points (the intersection points of $\beta_{v}$ with $\alpha_{j}$ and $\alpha_{j+1}$ ): two are white and two are gray. The white ones correspond to generators with $\epsilon(v)=+1$, the gray ones to those with $\epsilon(v)=-1$.

Of course, each Kauffman state is associated to $2^{\ell}$ different intersection points of $\mathbb{T}_{\alpha} \cap \mathbb{T}_{\beta}$ (where $\ell+1$ denotes the number of singular points), distinguished by their coordinates on the internal $\beta$-circles. More specifically, the intersection points in $\mathbb{T}_{\alpha} \cap$ $\mathbb{T}_{\beta}$ are in one-to-one correspondence with pairs $x$ a generalized Kauffman state and $\epsilon: s(P) \longrightarrow\{ \pm 1\}$, where here $s(P)$ denotes the set of singular points in the projection near which we have an internal $\beta$-circle (i.e. all but the distinguished one, $p$ ). Suppose that $(x, \epsilon)$ and $\left(x, \epsilon^{\prime}\right)$ represent two different intersection points $\mathbf{x}, \mathbf{x}^{\prime} \in \mathbb{T}_{\alpha} \cap \mathbb{T}_{\beta}$ with the same underlying Kauffman state, then we can find a homology class $\phi \in \pi_{2}\left(\mathbf{x}, \mathbf{x}^{\prime}\right)$ with $n_{\mathbf{w}}(\phi)=0$ and $2\left(n_{z_{v}^{1}}(\phi)+n_{z_{v}^{2}}(\phi)\right)=-\epsilon_{\mathbf{x}}(v)+\epsilon_{\mathbf{x}^{\prime}}(v)$. In fact, this homology class is a union of disjoint bigons. In particular, each Kauffman state $x$ is realized by a unique generator $\mathbf{x} \in \mathbb{T}_{\alpha} \cap \mathbb{T}_{\beta}$ which has maximal Alexander grading among all generators realizing $x$, corresponding to the map $\epsilon(v) \equiv+1$, for all $v$, cf. Figure 11 .

Our aim in this section is to establish the following precise version of Theorem 1.2:

Theorem 4.1. Fix a decorated projection for a singular knot $K$ with the property that the distinguished edge $Q$ is just below a singular point. Let $C_{d}(K, s)$ be the free Abelian group generated by generalized Kauffman states with Alexander grading s and Maslov grading $d$. There is a differential

$$
\partial: C(K, s) \longrightarrow C(K, s)
$$

which carries $C_{d}(K, s)$ to $C_{d-1}(K, s)$, with

$$
H_{d}\left(C_{*}(K, s), \partial\right) \cong \operatorname{HFS}_{d}(K, s) .
$$



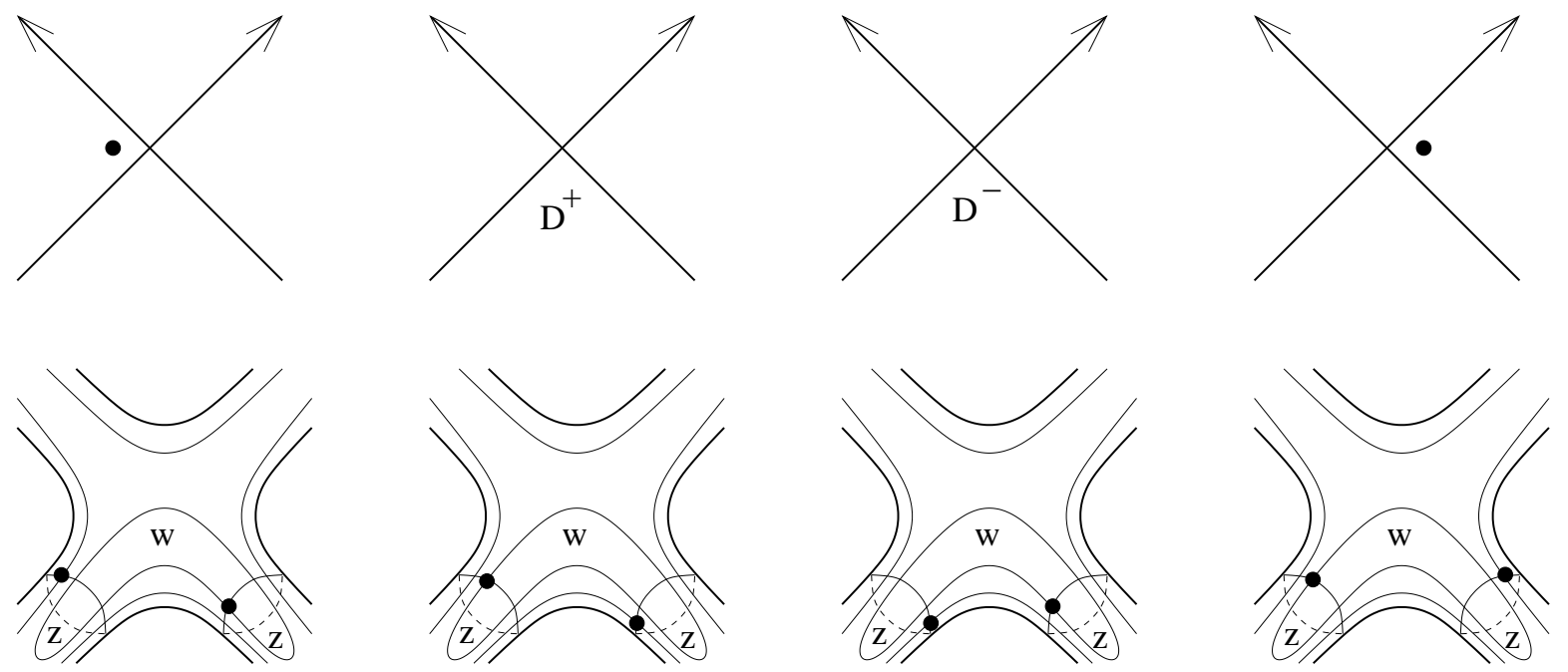

FiguRE 11. Alexander grading maximizers. For each Kauffman corner illustrated in the top row, we have drawn its corresponding Alexander grading maximizing intersection point below it.

Before giving the proof, we establish some lemmas. Indeed, we find it convenient to focus first on the case where $K$ is a planar singular link (which is in fact the case where Theorem 4.1 is the most valuable).

4.1. Planar singular links. We analyze the Heegaard diagram in the case where $K$ is a planar singular link (that is, admits an injective projection to the plane), establishing a special case of Theorem 4.1 in this case, from which Theorem 1.3 follows. First, we establish several lemmas.

Lemma 4.2. Let $K$ be a planar singular link. Let $x$ and $y$ be two generalized Kauffman states, and let $\mathbf{x}, \mathbf{y} \in \mathbb{T}_{\alpha} \cap \mathbb{T}_{\beta}$ unique Alexander grading maximizing intersection points representing them. Then, the difference between the Alexander gradings of $\mathbf{x}$ and $\mathbf{y}$ (in the sense of Equation (3)) coincides with the difference between the Alexander gradings $S(x)$ and $S(y)$ of the Kauffman states $x$ and $y$.

Proof. Given $x$ and $y$, it suffices to construct a particular homology class $\phi \in \pi_{2}(\mathbf{x}, \mathbf{y})$ which satisfies

$$
S(x)-S(y)=\sum_{i=1}^{\ell+1}\left(2 n_{w_{i}}(\phi)-n_{z_{i}^{1}}(\phi)-n_{z_{i}^{2}}(\phi)\right) .
$$

Indeed, it suffices to consider the case where there are no $D^{-}$corners in $x$ or $y$, in view of the following observation. Consider first the special case where $x$ and $y$ agree at all but $n$ corners, where $x$ is assigned $D^{+}$and $y$ is assigned $D^{-}$. In this case, it is easy to find 
a locally supported homology class $\phi \in \pi_{2}(\mathbf{x}, \mathbf{y})$ with $\sum n_{z_{i}}(\phi)=-n, \sum n_{w_{i}}(\phi) \equiv 0$, as illustrated in Figure 12.

Suppose then that $x$ and $y$ contain no $D^{-}$corners. We construct a curve $\gamma$ which connects $\mathbf{x}$ to $\mathbf{y}$, as in Figure 13. This curve $\gamma$ is a (possibly disconnected) closed path constructed from arcs within the $\alpha$ and $\beta$-circles. In fact, if $\mathbf{x}$ has components $x_{i}$ and $\mathbf{y}$ has components $y_{i}$, then $\gamma$ is constructed from arcs in $\alpha_{i}$ from $x_{i}$ to $y_{i}$ and arcs in $\beta_{j}$ from $y_{j}$ to $x_{k}$.

Now, order the edges $\left\{e_{i}\right\}$ in the order they are encountered in the singular knot, starting from $Q$. Let $v$ be some crossing where $e_{i}$ and $e_{i+1}$ meet. Fix reference points $T_{i}$ on the Heegaard diagram, placed on top of the $i^{\text {th }}$ edge, see the lower left picture in Figure 13. We can draw an arc $\epsilon_{i}$ from $T_{i}$ to $T_{i+1}$, which crosses only one of the $\alpha$-circle which is the meridian for the $i^{t h}$ edge, and none of the other circles. Similarly, let $\eta_{i}$ be the short arcs going from $w_{v}$ to $z_{v}^{1}$ and $z_{v}^{2}$ (inside the disk bounded by $\beta_{v}$ ). Then,

$$
\begin{aligned}
A(\mathbf{x})-A(\mathbf{y}) & =\sum \#\left(\eta_{i} \cap \partial \phi\right) \\
& =\sum \#\left(\left(\epsilon_{i}+\eta_{i}\right) \cap \partial \phi\right),
\end{aligned}
$$

since $\cup_{i} \epsilon_{i}$ is a closed curve. We claim that

$$
\#\left(\epsilon_{i}+\eta_{i}\right) \cap \partial \phi=S_{v}(x)-S_{v}(y)-\frac{1}{2}\left(W_{x(v)}(K)-W_{y(v)}(K)\right),
$$

where here $W_{r}(K)$ denotes the winding number of $K$ around a point in the region $r$. We see this as follows. The only part of $\partial \phi$ which intersects $\epsilon_{i}+\eta_{i}$ lies on the internal $\beta$-circle. Since the intersection number of $\epsilon_{i}+\eta_{i}$ with the internal $\beta$-circle is zero, it suffices to verify Equation (7) replacing $\partial \phi$ with any $\operatorname{arc}$ connecting $\mathbf{x}$ to $\mathbf{y}$ on the internal $\beta$-circle. Thus, we can verify Equation (7) by considering the various cases of $\mathbf{x}$ and $\mathbf{y}$ (locally, about each singular point), as illustrated in Figure 13.
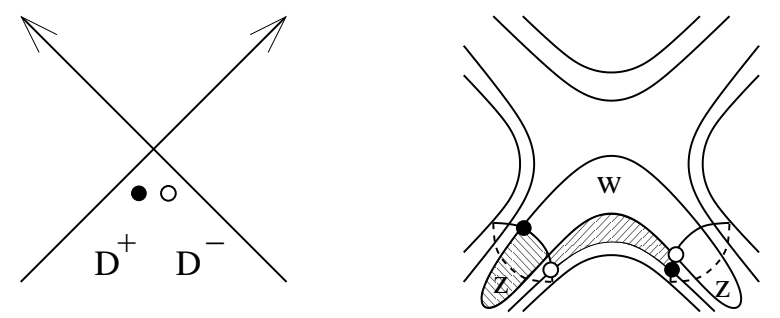

Figure 12. Domains for comparing $D^{+}$and $D^{-}$. We have illustrated on the right a domain $\phi$ which connects the Alexander grading maximizing generator of $D^{+}$with that of $D^{-}$. All multiplicities are +1 , -1 , or 0 ; regions with local multiplicity +1 are indicated by hatch marks from upper right to lower left, while regions with multiplicity -1 are indicated by the other hatching. 
Now, summing Equation (7), we see that

$$
A(\mathbf{x})-A(\mathbf{y})=\left(S(x)-\frac{1}{2} \sum_{v \in \operatorname{Cr}(P)} W_{x(v)}(K)\right)-\left(S(y)-\frac{1}{2} \sum_{v \in \operatorname{Cr}(P)} W_{y(v)}(K)\right) .
$$

But observe that

$$
\sum_{v \in \operatorname{Cr}(P)} W_{x(v)}(K)=\sum_{r \in R(P)-X-Y} W_{r}(K)
$$

is independent of the Kauffman state $\mathbf{x}$. This finishes the proof.

Definition 4.3. Let $K$ be a planar singular knot projection. Two generalized Kauffman states $x$ and $y$ are said to be equivalent if $x(v) \in\left\{A_{v}, D_{v}^{-}\right\}$if and only if $y(v) \in$ $\left\{A_{v}, D_{v}^{-}\right\}$. Let $x$ be a generalized Kauffman state. This determines an associated subgraph of the knot projection as follows. At each crossing $v$, if $x(v) \in\left\{A_{v}, D_{v}^{-}\right\}$, then we remove the lower left edge from the projection; if $x(v) \in\left\{C_{v}, D_{v}^{+}\right\}$, we remove the lower right edge from the crossing. We call the associated subgraph $\Gamma_{x}$ of the projection the pruning associated to the Kauffman state. Thus, two Kauffman states are equivalent if and only if they induce the same pruning.

Note that the pruning is a graph, with exactly one in-coming edge at each vertex, and at most two out-going ones.
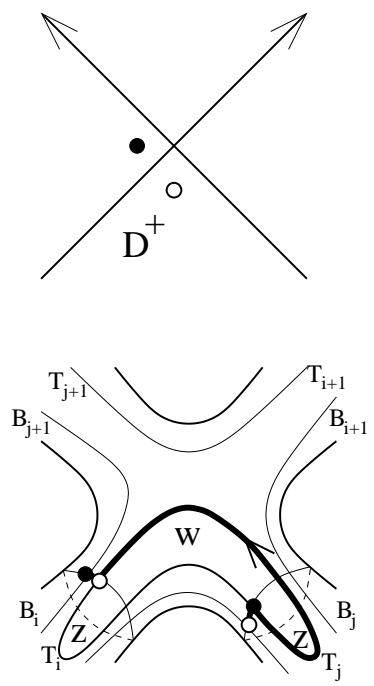
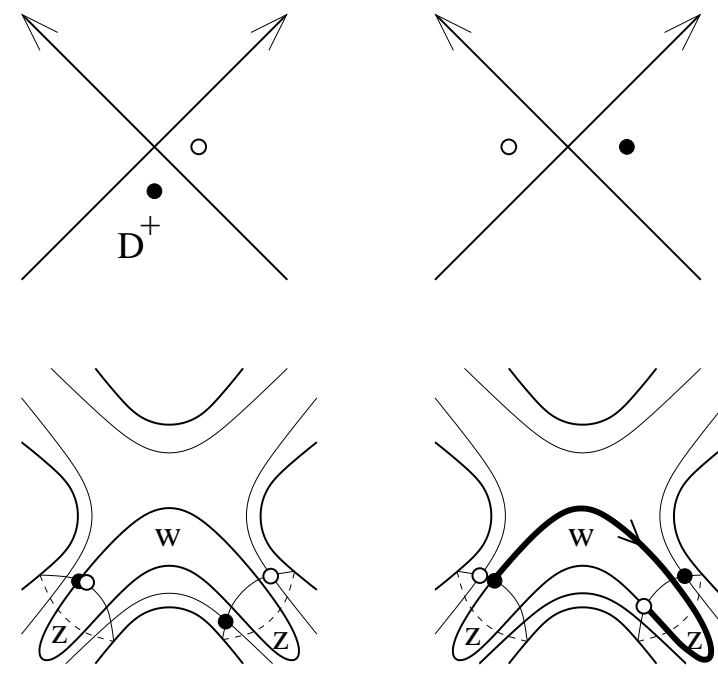

FIgURE 13. Verifying Equation (7). We verify that equation, using arcs connecting the black dot (representing $\mathbf{x}$ ) to the white dot (representing $\mathbf{y}$ ) supported on the internal $\beta$-circle. 
Lemma 4.4. The pruning associated to a Kauffman state for a planar singular knot is a connected graph.

Proof. Suppose that $\Gamma_{x}$ is not connected. We argue that $\Gamma_{x}$ must contain some cycle. This is seen by taking some edge $e$ which is in a different path component from the initial point, and continuing backwards through $e$. Since each vertex in $\Gamma_{x}$ has a unique incoming edge, this process can be continued; so the component through $e$ must contain a cycle.

Now, we consider some closed circuit $C$ in $\Gamma_{x}$ in a path component disjoint from the distinguished edge $Q$. The circuit $C$ can be thought of as enclosing a region $\Delta$ of the projection which does not contain the distinguished edge. We restrict our Kauffman state to $\Delta$. The restriction of our projection to $\Delta$ gives the region, which is topologically a disk, the structure of a $C W$ complex, where the vertices correspond to the $V$ crossings, and edges corresponding to the $E$ arcs in $\Delta$, and which divide $\Delta$ into $F$ faces. There are four kinds of vertices in the one-complex of $\Delta$ : bivalent ones (with one in-coming and one out-going edge) whose number is $D$, vertices with two in-coming and one out-going edge whose number is $T_{1}$, vertices with one in-coming and two out-going edges whose number is $T_{2}$, and four-valent ones (two in-coming, and two out-going edges) whose number is $W$, so that $V=D+T_{1}+T_{2}+W$. Note that all vertices counted in $D, T_{1}$, and $T_{2}$ occur on the boundary of $\Delta$. By counting edges, we can verify that $T_{1}=T_{2}$. In fact, the total number of edges is given by $E=D+3 T_{2}+2 W$. But since $\Delta$ is a disk, its Euler characteristic is one, so we get the relation that $F-T_{2}-W=1$. Since each face in $\Delta$ is occupied by a Kauffman corner (we are using here the fact that $Q$ is not contained in $\Delta$ ), the restriction of our Kauffman state $x$ to $\Delta$ demonstrates that $F \leq T_{1}+W$ (at each vertex $v$ counted in $D$ or $T_{2}, x(v)$ is not a face of $\Delta$, whereas at each vertex $v$ in $W, x(v)$ is a face of $\Delta$, while at each vertex $v$ in $T_{1}, x(v)$ might or might not be a face of $\Delta$ ), contradicting $T_{1}=T_{2}$ and $F-T_{2}-W=1$.

This has the following easy consequence:

Lemma 4.5. If $x$ and $y$ are generalized Kauffman states for a planar singular knot $K$ which are equivalent, then $x$ and $y$ coincide.

Proof. We construct the following subset $P$ of the complement of $\Gamma_{x}$. Suppose that $x$ and $y$ differ at a crossing $v$. Then, of course there is a different crossing $w$ with the property that $y(w)$ and $x(v)$ are assigned to the same region. We then connect $y(w)$ and $x(v)$ along some arc in the complement of the knot projection. Next, we connect $x(v)$ to $y(v)$ by an arc which crosses one of the four edges of our knot projection; but that is precisely the edge removed to obtain $\Gamma_{x}$. We continue this procedure.

In this manner, we construct a collection of closed curves $P$. If $P$ is non-empty, let $R$ be any connected component of $P$. It is easy to see that $R$ divides the plane into 
two regions, both of which contain points in $\Gamma_{x}$. But this contradicts the fact that (by Lemma 4.4) $\Gamma_{x}$ is connected.

We now have the ingredients required to establish Theorem 4.1, at least in the case when $K$ is a planar singular link.

Proposition 4.6. Theorem 4.1 holds for planar singular links; i.e. if $K$ is a planar singular link, let $C_{d}(K, s)$ be the free Abelian group generated by generalized Kauffman states with Alexander grading $s$ and Maslov grading d. There is a differential

$$
\partial: C(K, s) \longrightarrow C(K, s)
$$

which carries $C_{d}(K, s)$ to $C_{d-1}(K, s)$, with

$$
H_{d}\left(C_{*}(K, s), \partial\right) \cong \operatorname{HFS}_{d}(K, s) .
$$

Proof. If $\epsilon$ and $\epsilon^{\prime}$ differ at a single singular point $v$, then the homology class $\phi$ clearly admits a single holomorphic representative up to reparametrization. In fact, by placing basepoints at all the other regions of our Heegaard diagram, we obtain a filtration of the chain complex CFS whose $E_{0}$ term counts only these short differentials; i.e. its differentials are given by tensor product of the space of Kauffman states with $\ell$ chain compexes of the form

$$
\mathbb{F}\left[U_{1}, \ldots, U_{\ell}\right] \stackrel{U_{i}}{\longrightarrow} \mathbb{F}\left[U_{1}, \ldots, U_{\ell}\right]
$$

It is easy to see that the $E_{1}$ term, now, is simply the free $\mathbb{F}$-module generated by the Kauffman states. Indeed, this homology is carried by the pairs $\left(x, \epsilon^{-}\right)$, where $\epsilon^{-}(v)=-1$ for each $v$.

It remains to identify the Alexander and Maslov gradings of the intersection points $\left(x, \epsilon^{-}\right)$with the corresponding gradings for their underlying Kauffman states. This statement for the Alexander grading is an immediate consequence of Lemma 4.2. For the statement about the Maslov gradings, it suffices to show that the algebraic grading $N$ of any of the Alexander grading maximizing generators vanishes identically. To this end, let $\mathbf{x}$ be a generator and $x$ its underlying Kauffman state. We claim that there is a new Heegaard diagram, obtained by moving the central $\beta$-circles, each across exactly one of the two $z_{j}^{i}$ for fixed $j$, so as to cancel two of the intersection points of this $\beta$ with one of the two $\alpha$-circle meridians, depending on the value of $x(v)$, as illustrated in Figure 14. Let $\gamma_{v}$ denote the isotoped image of $\beta_{v}$. Note that the $\gamma_{v}$ are chosen so that the intersection point $\mathbf{x}$ persists into the new diagram. Indeed, the generators for the new diagram now correspond to pairs $(y, \epsilon)$, where here $y$ is some Kauffman state equivalent to $x$, and $\epsilon: s(P) \longrightarrow\{ \pm 1\}$. According to Lemma 4.5 it follows at once that the generators for the new diagram correspond simply to maps $\epsilon: s(P) \longrightarrow\{ \pm 1\}$, since they all have the same underlying Kauffman state. We claim that this is an admissible Heegaard diagram for $S^{3}$ with $\ell$ basepoints (supplied by $\mathbf{w}$ ). To see admissibility, we proceed as follows. Let $\Pi$ be a periodic domain with multiplicity $m$ near some singular 

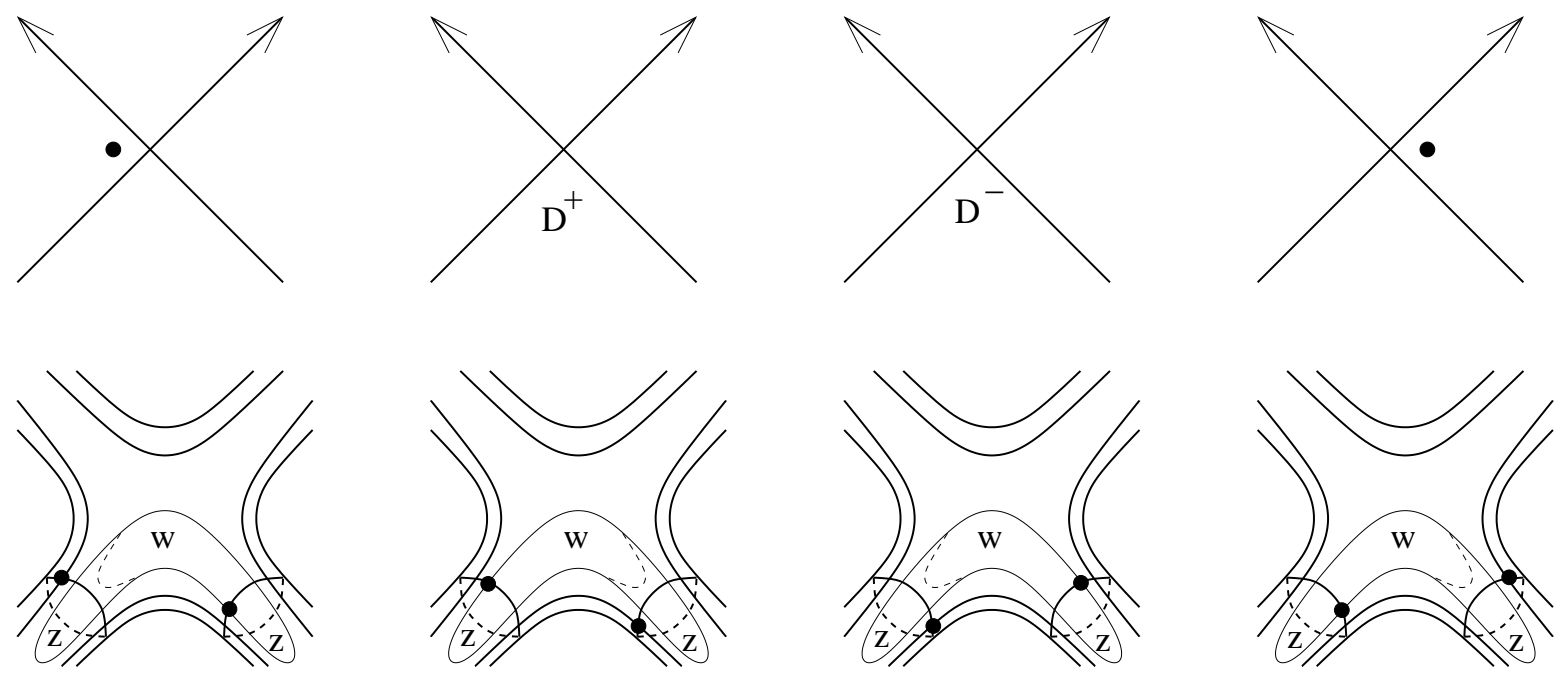

FiguRE 14. Isotopies of the standard diagram. Given the generator $\mathbf{x}$, we consider the new diagram obtained by isotoping the central $\beta$-circle as indicated by the light dotted line. Note that the new diagram for $A$ and $D^{-}$coincide, while the diagram for $C$ and $D^{+}$coincide.

point $v$. Let $u$ be the preceding vertex to $v$ in the pruning $\Gamma_{x}$. If $\Pi$ has only nonnegative multiplicities, then its local multiplicity near $u$ must be greater than or equal to $m$. Iterating this, we can go back to the root of the pruning, in view of Lemma 4.4, where the local multiplicity in turn must equal zero. This shows that our periodic domain must vanish identically.

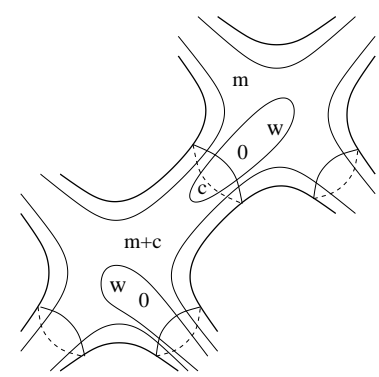

FIgURE 15. Verifying admissibility. We have illustrated here the Heegaard diagram near two vertices, along with part of a periodic domain. (We have suppressed the basepoints $z$, as they no longer play a role.) If the periodic domain has local multiplicity $m$ near the vertex $v$, and local multiplicity $c$ in the small region, then its local multiplicity near the preceding vertex $u$ in the pruning must be $m+c$. 
Next, note that the isotopy, of course, changes the Floer homology; but it leaves invariant the theory where all $U_{i}=1$ for $i>1$ (inducing an isomorphism between $N$-graded theories). Indeed, recall [9] that the isomorphism induced on homology by isotopies can be thought of as induced by a chain map which counts holomorphic triangles. Moreover, the count of holomorphic triangles clearly takes the generator corresponding to $(x, \epsilon)$, to the generator corresponding to the same $\epsilon$ (respecting $N$-gradings). The result follows.

This immediately gives a proof of Theorem 1.3:

Proof. [of Theorem 1.3.] Applying Proposition 4.6, we see that the differential actually must vanish identically, since the state sum ensures that for fixed Alexander grading, the generators of the chain complex have fixed Maslov grading.

4.2. The general case of Theorem 4.1. The discussion above can be generalized to the case of (non-planar) singular knots, as well. For example, we have the following generalization of Lemma 4.2:

Lemma 4.7. Let $K$ be a singular link. Let $x$ and $y$ be two generalized Kauffman states, and let $\mathbf{x}, \mathbf{y} \in \mathbb{T}_{\alpha} \cap \mathbb{T}_{\beta}$ unique Alexander grading maximizing intersection points representing them. Then, the difference between the Alexander gradings of $\mathbf{x}$ and $\mathbf{y}$ (in the sense of Equation (3)) coincides with the difference between the Alexander gradings $S(x)$ and $S(y)$ of Kauffman states $x$ and $y$.

Proof. We argue as in the proof of Lemma 4.2, establishing Equation (7) in the presence of non-singular intersection points. This time, it is the central $\alpha$-circle which meets the curve $\cup \epsilon_{i}$. Its total intersection number with this curve is zero, so it sufficies to verify Equation (7) by replacing $\partial \phi$ with arcs in the central $\alpha$-circle which connect the two generators $\mathbf{x}$ and $\mathbf{y}$, compare Figure 16. This is straightforward to verify, and the previous proof goes through.

Proof. [of Theorem 4.1] Proceed as in the proof of Proposition 4.6 to see that there is a chain complex generated by Kauffman states. Lemma 4.7 verifies the identification between the Alexander gradings coming from the Heegaard diagram with the state sum formula.

It remains to verify that the Maslov grading for each Alexander grading maximizing generator $\mathbf{x}$ corresponding to a given Kauffman state $x$ is given by the state sum formula; indeed, since we have already verified the corresponding statement for the Alexander gradings, it suffices to verify the corresponding statement for the $N$-grading (which has the advantage that it is independent of the placement of the $z_{i}$ basepoints). Proceeding as before (as indicated in Figure 14), we isotope $\beta_{v}$ across one of the basepoints $z_{j}^{i}$ at 

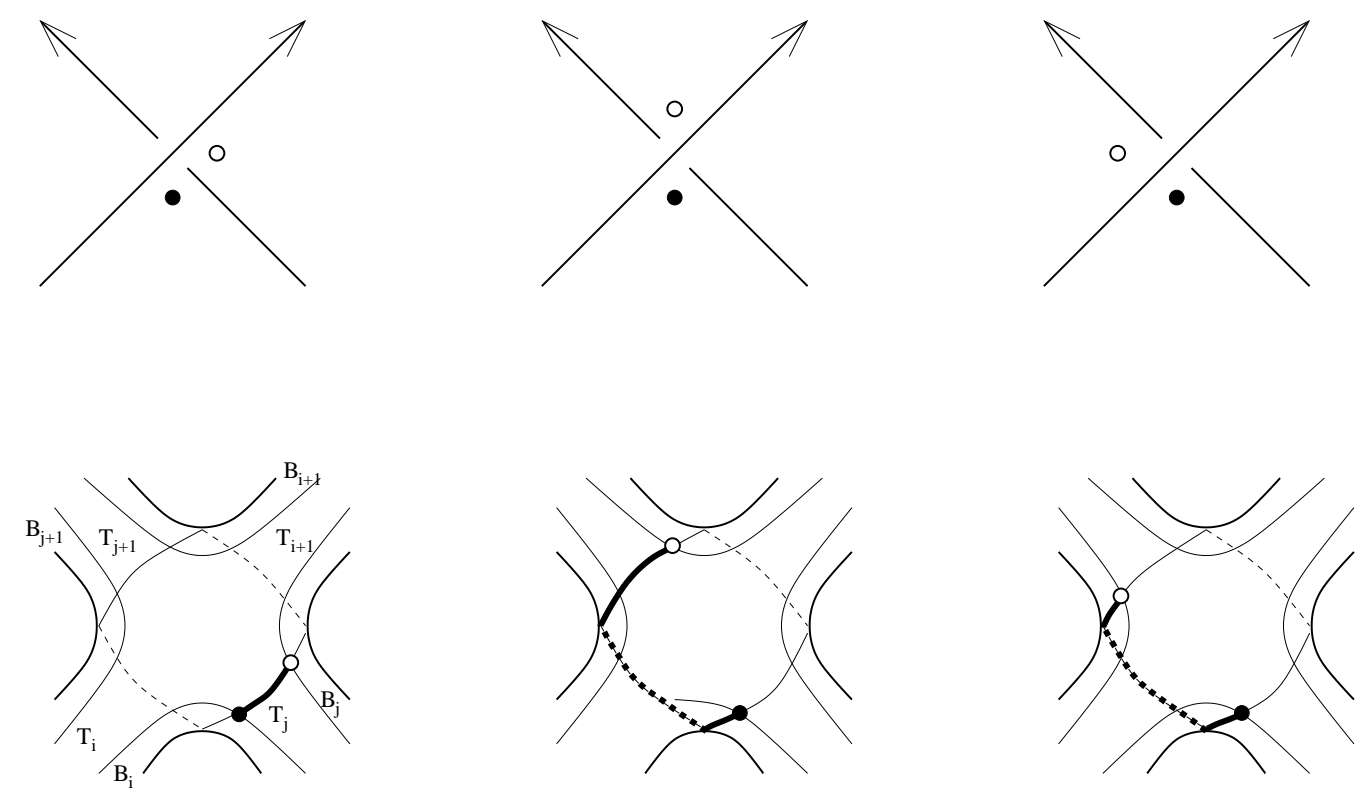

FIguRE 16. Verifying Equation (7) in the presence of nonsingular crossings. The black dot represents $\mathbf{x}$, the white represents y. For negative crossings, rotate the diagram.

each singular crossing $v$ to get a new circle. In fact, we can equivalently view this as an isotopy of one of the $\alpha$-circles intersecting $\beta_{v}$, replacing it with a new circle $\gamma_{v}$ disjoint from $\beta_{v}$. Consider next a non-singular crossing $v$. If $x(v)$ is of type $A$ or $C$, we replace the $\alpha$-circle by a new circle $\gamma_{v}$ which is a meridian for the corresponding in-coming edge. If $x(v)$ is of type $D$, we replace its corresponding $\beta$-circle by the meridian $\gamma_{v}$ for either of the two in-coming edges. Finally if $x(v)$ is of type $B$, we replace the $\alpha$-circle by a new circle $\gamma_{v}$ supported locally near the crossing pictured, as shown by Figure 17.

In this manner, we obtain a new $\operatorname{diagram}(\Sigma, \boldsymbol{\gamma}, \boldsymbol{\beta}, \mathbf{w}, \mathbf{z})$, equipped with a set $S=$ $\mathbb{T}_{\gamma} \cap \mathbb{T}_{\beta}$, from which we have a map to the Kauffman states for $K$. Indeed, elements of $S$ map to the Kauffman states for the projection of another knot $K_{0}$ which is obtained by resolving all the crossings where $x(v)=B$. Let $x_{0}$ denote the induced Kauffman state on $K_{0}$. It is easy to see that any other intersection point $\mathbf{y}$ of $\mathbb{T}_{\gamma} \cap \mathbb{T}_{\beta}$ induces a Kauffman state $y$ which associates $B$ or $D$ to each vertex $v$ where $x(v)=B$, and hence it can be restricted to $K_{0}$. In fact, $y_{0}$ is equivalent to $x_{0}$, and hence by Lemma 4.5 $x_{0}=y_{0}$. From this, it follows easily that $x=y$. Indeed, Lemma 4.4 shows that the $\gamma$ circles divide $\Sigma$ into $\ell+1$ components. Since the span of the $\gamma_{i}$ is contained in the span of the $\alpha_{i}$, it follows that these two spans coincide, and that $(\Sigma, \boldsymbol{\gamma}, \boldsymbol{\beta}, \mathbf{w})$ is a Heegaard diagram for $S^{3}$.

Now, given generator $\mathbf{x} \in \mathbb{T}_{\alpha} \cap \mathbb{T}_{\beta}$ (maximizing Alexander grading among all intersection points corresponding to the Kauffman state $x$ ), we have exhibited a new 

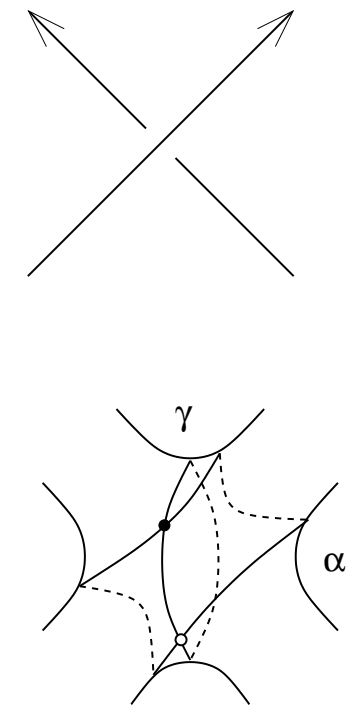
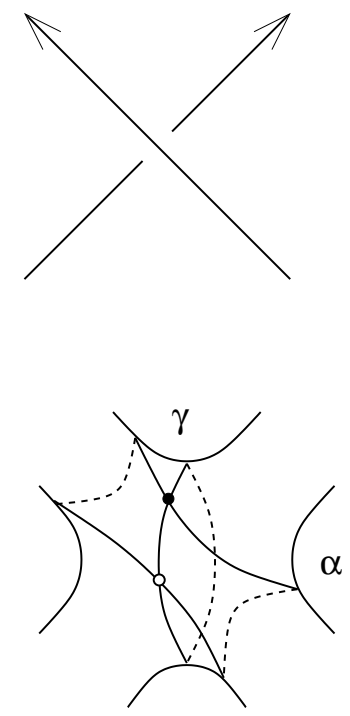

Figure 17. A generator $\Theta^{\prime}$. The black dots represent the generator $\Theta^{\prime} \in \mathbb{T}_{\gamma} \cap \mathbb{T}_{\alpha}$. At each vertex, the $\gamma$ - and $\alpha$-circles meet in two points, one of which we colored black, the other white. For a positive crossing, the black has $N$-grading one greater than the white generator; this can be verified using the obvious bigon (minus disks) going from black to white. Similarly, at a negative crossing, the $N$-grading of the white generator is one greater than the $N$-grading of the black one.

Heegaard diagram, equipped with a corresponding Alexander grading maximizing generator $\mathbf{x}^{\prime} \in \mathbb{T}_{\alpha} \cap \mathbb{T}_{\gamma}$, which clearly has $N$-grading equal to zero. In fact, $\mathbb{T}_{\alpha} \cap \mathbb{T}_{\gamma}$ also has a minimal number of intersection points. Let $\Theta$ denote its $N$-maximizing generator. If $n$ denotes the number of negative crossings $v$ where $x(v)=B$ (and $x$ is the Kauffman state corresponding to $\mathbf{x}$ ), and $p$ denotes the number of such positive crossings, then we claim that there is an obvious $\psi \in \pi_{2}\left(\Theta^{\prime}, \mathbf{x}, \mathbf{x}^{\prime}\right)$ where $\operatorname{gr}\left(\Theta^{\prime}\right)=\operatorname{gr}(\Theta)-n$, and $\mu(\psi)=-p$, cf. Figure 18. The map induced by counting holomorphic triangles preserves gradings, in the sense that $N\left(\Theta^{\prime}\right)+N(\mathbf{x})-\mu(\psi)=N\left(\mathbf{x}^{\prime}\right)$, from which it follows now that $N(\mathbf{x})=n-p$. Comparing with Figures 8 and 9 , we have verified that $N(\mathbf{x})=M(x)-2 S(x)$.

The proof of Theorem 1.1 is an immediate corollary of Theorem 4.1:

Proof. [of Theorem 1.1.] Equation (2) is an immediate consequence of Theorem 4.1 and Proposition 3.2.

Equation (1) follows by a simple comparison of the chain complexes: each generator $\mathbf{x}$ for $\widetilde{\mathrm{CFS}}$ corresponds infinitely many generators for $U_{1}^{n_{1}} \cdot \ldots \cdot U_{\ell}^{n_{\ell}}$ indexed by $\ell$-tuples 

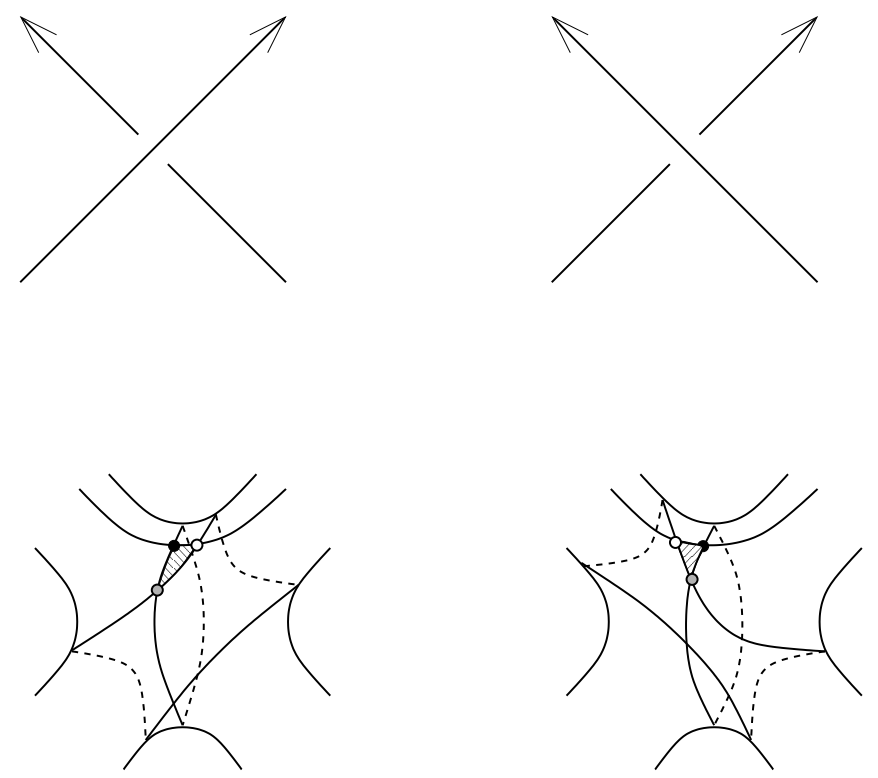

FiguRE 18. Triangles. We denote the generator $\Theta^{\prime}$ by a grey dot; $\mathbf{x}^{\prime} \in \mathbb{T}_{\alpha} \cap \mathbb{T}_{\gamma}$ and $\mathbf{x} \in \mathbb{T}_{\alpha} \cap \mathbb{T}_{\beta}$. The triangle on the left (corresponding to the positive crossing) has local coefficent -1 (and indeed Maslov index equal to -1 ) while the one on the right has positive local multiplicity (and Maslov index equal to zero).

of non-negative integers $\left(n_{1}, \ldots, n_{\ell}\right)$. Each such generator occupies Alexander grading $A(\mathbf{x})-\sum_{k=1}^{\ell} n_{k}$ and $N$-grading equal to the $N$-grading of x. Equation (1) follows.

According to Theorem 1.3, calculating $\operatorname{HFS}(K)$ for a planar knot is equivalent to computing its Alexander polynomial. This can be efficiently done either using the state sum formula

$$
\Delta_{K}(T)=\sum_{x \in K(P)} \prod_{v \in \operatorname{Cr}(P))} Z_{v}(x)
$$

we have discussed in Section 3, or the skein relations

$$
\begin{aligned}
& \Delta_{K^{+}}(T)=\Delta_{K}(T)+T^{\frac{1}{2}} \cdot \Delta_{K^{o}}(T) \\
& \Delta_{K^{-}}(T)=\Delta_{K}(T)+T^{-\frac{1}{2}} \cdot \Delta_{K^{o}}(T),
\end{aligned}
$$

we have used in the definition of the Alexander polynomial for singular links.

\section{Some calculations}

5.1. Planar singular links. Recall that a singular link $K$ is called planar if it admits an injective projection to the plane. For such links a simple Heegaard diagram of genus 
zero can be given in the following way. Fix a planar singular link $K$, consider an injective projection, contract all its thick edges to singular points and take the $\alpha$ - and $\beta$-curves at every crossing as it is instructed by Figure 19. Note that an $\alpha$-curve corresponds to each thick edge and the two outgoing thin edges, while a $\beta$-curve corresponds to a thick edge and two incoming thin edges. By taking these curves for all the crossings and then deleting an (arbitrary) $\alpha$ - and $\beta$-curve from the collection, we get a Heegaard diagram of a singular link.

Proposition 5.1. The resulting Heegaard diagram is compatible with the given planar singular link $K$.

We can distinguish coordinates of an intersection point $\mathbf{x}=\left(x_{1}, \ldots, x_{\ell}\right) \in \mathbb{T}_{\alpha} \cap \mathbb{T}_{\beta}$ according to whether $x_{i}$ corresponds to a thin or thick edge — in the diagram it is reflected by the fact whether $x_{i}$ is near a base point of type $\mathbf{z}$ or of type $\mathbf{w}$. Since near a fixed $w$ or $z$ the $\alpha$ - and $\beta$-curves intersect each other in two points, we can group our intersection points into groups of cardinality $2^{\ell}$.

5.2. An example. We will illustrate the above principle by an example. This example also shows that the Floer homology theory $\widehat{\mathrm{HFS}}$ is not determined naively by the Alexander polynomial of a planar singular knot.

By taking the $(3,3)$ torus link and singularizing its natural projection we get the singular knot $K$ depicted by Figure 20. The planar Heegaard diagram corresponding to this projection is shown by Figure 21. The diagram also indicates (with dashed lines) the $\alpha$ - and $\beta$-curves which we delete according to the algorithm for constructing the diagram from the projection.

The Alexander polynomial of the singular knot $K$ given above can easily be computed from the state sum formula, giving

$$
\Delta_{K}(T)=T^{2}+5 \cdot T+9+5 \cdot T^{-1}+T^{-2} .
$$
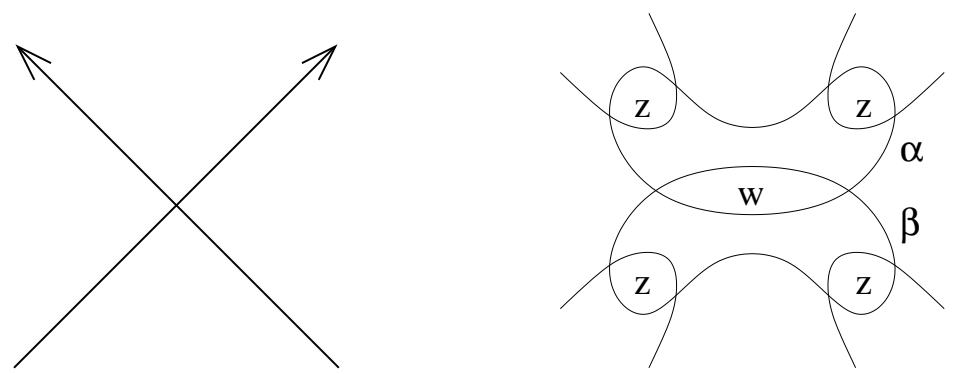

Figure 19. Heegaard diagram of a planar singular link. The crossing on the left (obtained by contracting a thick edge) is replaced by the piece of Heegaard diagram on the right. 


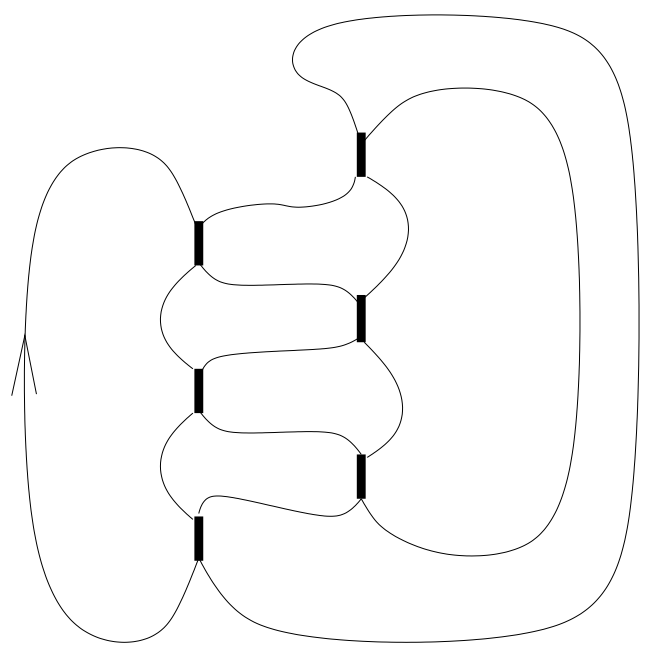

Figure 20. Singular knot given as the singularization of the projection of the $(3,3)$ torus link.

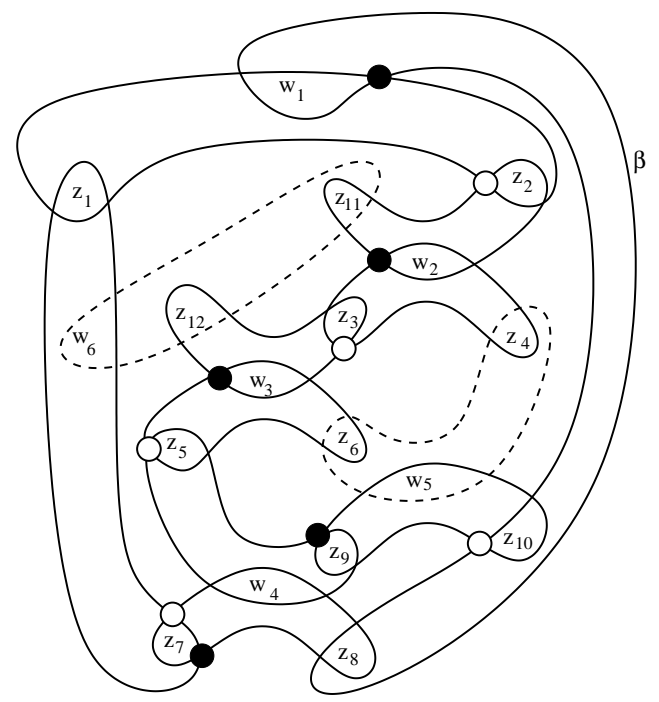

FigURE 21. The planar Heegaard diagram corresponding to the singular knot projection of Figure 20.

It follows from Theorem 1.1 that

$$
\sum_{s, d}(-1)^{d} \text { rk } \widetilde{\operatorname{HFS}_{d}}(K, s) \cdot T^{s}=-T^{7}+6 \cdot T^{5}-21 \cdot T^{3}+21 \cdot T^{2}-6+T^{-2}
$$


In the following we will identify the generators of the Floer chain complex $\widetilde{\mathrm{CFS}}$ and determine part of the boundary map $\widetilde{\partial}$ for this particular singular knot. In certain Alexander gradings, this differential is trivial; indeed, we have the following:

Proposition 5.2. The rank of $\widetilde{\operatorname{HFS}}(K, s)$ for $s=2,3$ is 21 , for $s=0,5$, it is 6 , for $s=-1,6$ is 0 while for $s=-2,7$ it is 1 .

Proof. This is a straightforward calculation, explicitly identifying the generators, and finding some boundary maps. See the proof of Proposition 5.3 for more details.

The rank of $\widetilde{\mathrm{HFS}}$ is not equal to its Euler characteristic. More precisely we show that for the above singular link $K$

Proposition 5.3. The Floer homology group $\widetilde{\operatorname{HFS}}_{*}(K, 4)$ is nontrivial, although its Euler characteristic is zero.

Proof. In order to prove this statement, we first list the intersection points of the tori $\mathbb{T}_{\alpha}$ and $\mathbb{T}_{\beta}$ in $S y m^{5}\left(S^{2}\right)$ and identify the ones which have Alexander grading 4 . First of all notice that there are four types of intersection points: the points of type $A$ consist of those intersection points which have coordinates near $\left(w_{2}, w_{3}, w_{4}, z_{1}, z_{10}\right)$; for type $B$ points this vector is $\left(z_{2}, z_{3}, z_{5}, z_{7}, z_{10}\right)$, for type $C$ points this vector is $\left(w_{2}, w_{3}, z_{1}, z_{8}, z_{9}\right)$, and finally for type $D$ points this vector is $\left(w_{1}, w_{2}, w_{3}, z_{7}, z_{9}\right)$. The two possible coordinates near a base point $z_{i}$ (or $w_{j}$ ) will be distinguished based on the property whether it is to the left or to the right from the edge corresponding to the base point (when using the orientation opposite of the vertex). Near a $w$-type point the two choices will be denoted by $L$ and $R$, near a $z$-type point by $l$ and $r$. We also keep the order of listing $w$ 's first (with increasing indices) followed by $z$ 's (also with increasing indices). For example, the intersection point $\mathbf{x}_{1} \in \mathbb{T}_{\alpha} \cap \mathbb{T}_{\beta}$ indicated by the heavy dots in Figure 21 is represented by $D(R, L, L, r, l)$, while the light dots $\mathbf{y} \in \mathbb{T}_{\alpha} \cap \mathbb{T}_{\beta}$ in the same figure is represented by $B(l, r, l, l, l)$.

It is easy to see that changing $L$ to $R$ drops the $N$-grading by 1 and raises the Alexander grading by 2, while a change of $l$ to $r$ drops the $N$-grading by 1 and raises the Alexander grading by 1 . For example, the highest Alexander grading is attained by the intersection point $D(L, L, L, l, l)$ while the lowest Alexander grading is taken by $A(R, R, R, r, r)$.

The above combinatorial computation shows that there are twenty intersection points with Alexander grading 4, ten of which have (up to a suitable translation) Maslov grading 2 and ten have Maslov grading 1.

Consider the following eleven intersection points: $\mathbf{x}_{1}=D(R, L, L, r, l)$ and $\mathbf{x}_{2}=$ $B(l, r, l, l, l)$ (depicted in Figure 21 by the heavy and light circles), and also $\mathbf{b}_{1}=$ $B(r, l, l, l, l), \mathbf{b}_{3}=B(l, l, r, l, l), \mathbf{b}_{4}=B(l, l, l, r, l), \mathbf{b}_{5}=B(l, l, l, l, r)$, and $\mathbf{a}_{3}=A(L, L, R, l, l)$ 
(of Maslov grading 1); and $\mathbf{D}_{2}=D(L, R, L, r, l), \mathbf{D}_{3}=D(L, L, R, r, l), \mathbf{D}_{5}=D(L, R, L, l, r)$, and $\mathbf{D}_{6}=D(L, L, R, l, r)$ (of Maslov grading 2). (In this notation $\mathbf{x}_{1}=\mathbf{D}_{1}$, and $\mathbf{x}_{2}=\mathbf{b}_{2}$.) There are nine nonnegative homotopy classes with $n_{\mathbf{z}}=n_{\mathbf{w}}=0$ connecting nine of the above eleven intersection points $\mathbf{b}_{i}(i \neq 2), \mathbf{D}_{j}(j \neq 1)$ and $\mathbf{a}_{3}$ to the remaining nine intersection points of Alexander grading 4. It is easy to see that the contribution of each of these nine homotopy classes in the boundary map is equal to \pm 1 . Next we will show that there are no more pairs of intersection points among the eleven above with the property that a homotopy class connecting them with $n_{\mathbf{z}}=n_{\mathbf{w}}=0$ exists. Clearly, this fact implies that there are no more boundary maps we should take into account when computing $\widetilde{\operatorname{HFS}}(K, 4)$, verifying that $\widetilde{\operatorname{HFS}}(K, 4)=\mathbb{F} \oplus \mathbb{F}$ (with $\mathbb{F}=\mathbb{Z} / 2 \mathbb{Z})$, concluding our computation.

For the last claim about the nonexistence of further homotopy classes with $n_{\mathbf{z}}=$ $n_{\mathbf{w}}=0$ we argue as follows. We find domains from $\mathbf{x}_{1}$ to the other ten distinguished intersection points $\left\{\mathbf{b}_{1}, \mathbf{x}_{2}, \mathbf{b}_{3}, \mathbf{b}_{4}, \mathbf{b}_{5}, \mathbf{a}_{3}, \mathbf{D}_{2}, \mathbf{D}_{3}, \mathbf{D}_{5}, \mathbf{D}_{6}\right\}$ (for example, the ones pictured in Figure 22). Associate to each such domain $\mathcal{D}$ the corresponding 18-dimensional vector $\left(n_{\mathbf{z}}(\mathcal{D}), n_{\mathbf{w}}(\mathcal{D})\right)$. (Recall that there are 12 points of type $z$ and 6 of type $w$.) This 18-dimensional vector space contains a subspace $V$ generated by the vectors $\left(n_{\mathbf{z}}(\mathcal{P}), n_{\mathbf{w}}(\mathcal{P})\right)$, where $\mathcal{P}$ runs over all domains with boundary among the $\alpha_{i}$ and $\beta_{j}$. Our claim amounts to showing that the original 10 vectors, together with the additional zero vector, are distinct modulo $V$. To this end, consider functions $F_{1}=w_{1}^{*}-z_{1}^{*}-z_{8}^{*}+z_{7}^{*}$,
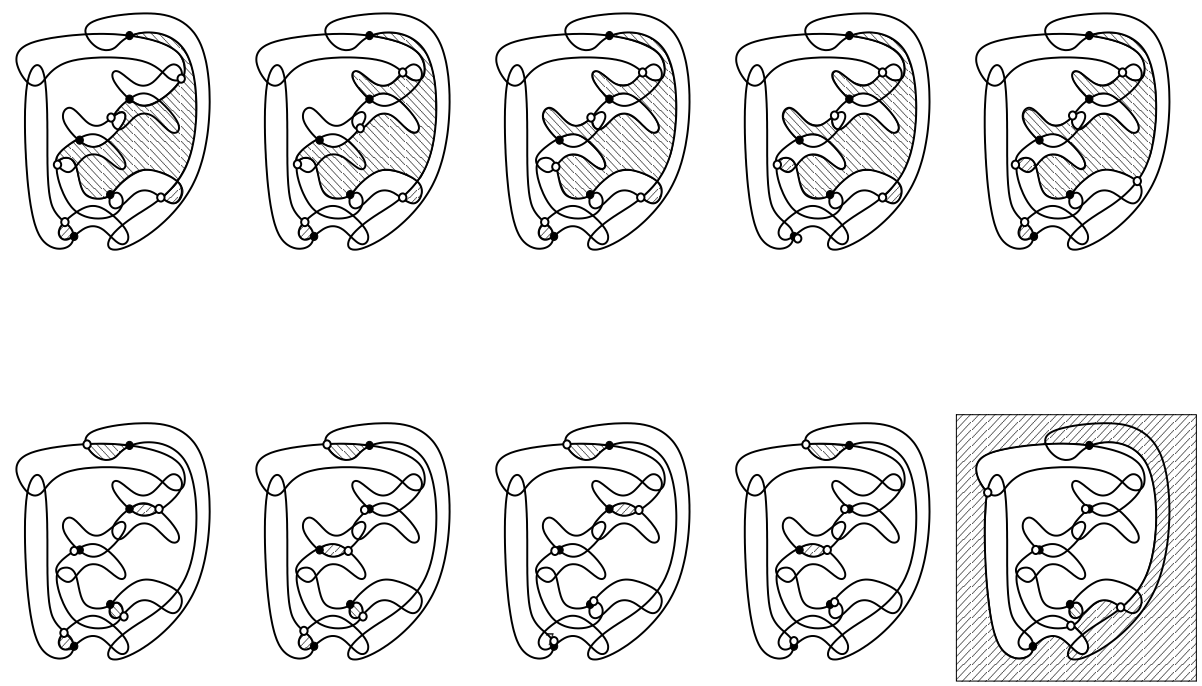

Figure 22. Domains. We depict domains from $\mathbf{x}_{1}$ to the other 10 distinguished generators (in order: $\mathbf{b}_{1}, \mathbf{x}_{2}, \mathbf{b}_{3}, \mathbf{b}_{4}, \mathbf{b}_{5}, \mathbf{D}_{5}, \mathbf{D}_{6}, \mathbf{D}_{2}, \mathbf{D}_{3}$, $\mathbf{a}_{3}$ ). The generator $\mathbf{x}_{1}$ is denoted by the black dots. Local multiplicities are $0,-1$ (denoted by hatchings in one direction), and +1 (hatchings in the other direction). 
$F_{2}=w_{2}^{*}-z_{3}^{*}-z_{11}^{*}+z_{12}^{*}, F_{3}=w_{3}^{*}-z_{6}^{*}-z_{3}^{*}+z_{4}^{*}, F_{4}=w_{4}^{*}-z_{8}^{*}-z_{9}^{*}+z_{10}^{*}, F_{5}=w_{5}^{*}-z_{6}^{*}-z_{9}^{*}+z_{5}^{*}$, which can be easily shown to vanish on $V$. It is straightforward to verify that these five functions take on 11 distinct values on the 11 vectors. (For the sake of completeness, we list the vectors of our chosen domains connecting $\mathbf{x}_{1}$ to the points in the obvious basis of $\mathbb{Z}^{18}$, where the basis vectors are identified with the $z_{i}$ 's and the $w_{j}$ 's: for $\mathbf{b}_{1}$ it is $\left(z_{4}+z_{6}-z_{7}-z_{10}\right), \mathbf{b}_{2}$ is $\left(z_{6}-z_{7}-z_{10}+z_{11}\right), \mathbf{b}_{3}$ is $\left(-z_{7}-z_{10}+z_{11}+z_{12}\right), \mathbf{b}_{4}$ is $\left(-z_{5}-z_{10}+z_{11}+z_{12}\right), \mathbf{b}_{5}=-z_{5}-z_{7}+z_{11}+z_{12}, \mathbf{D}_{5}$ is $-z_{7}+z_{9}+w_{1}-w_{2}, \mathbf{D}_{6}$ is $-z_{7}+z_{9}+w_{1}-w_{3}, \mathbf{D}_{2}$ is $w_{1}-w_{2}, \mathbf{D}_{3}$ is $w_{1}-w_{3}, \mathbf{a}_{3}$ is $\left.z_{9}-z_{10}.\right)$

We conclude that $\widehat{\operatorname{HFS}}(K, 4)$ is generated by the two generators $\mathbf{x}_{1}=D(R, L, L, r, l)$ and $\mathbf{x}_{2}=B(l, r, l, l, l)$ depicted in Figure 21 by the heavy and light circles. Consequently we see that

$$
\widetilde{\operatorname{HFS}}(K, 4) \cong \mathbb{F} \oplus \mathbb{F},
$$

showing that the total rank of the Floer homology of $\widetilde{\text { HFS }}$ can exceed the absolute value if its Euler characteristic.

Note that a similar calculation can be performed for $s=1$, showing that the corresponding Floer homology group is also isomorphic to $\mathbb{F} \oplus \mathbb{F}$.

\section{REFERENCES}

[1] B. Audoux. Heegaard-Floer homology for singular links. arXiv:0705.2377.

[2] L. H. Kauffman. Formal knot theory. Number 30 in Mathematical Notes. Princeton University Press, 1983.

[3] M. Khovanov and L. Rozansky. Matrix factorizations and link homology. Fund. Math., 199(1):1-91, 2008.

[4] H. Murakami, T. Ohtsuki, and S. Yamada. Homfly polynomial via an invariants of colored plane graphs. Enseign. Math., 44:325-360, 1998.

[5] P. S. Ozsváth and Z. Szabó. A cube of resolutions for knot Floer homology. arXiv:0705.3852, To appear J. Topology.

[6] P. S. Ozsváth and Z. Szabó. Heegaard Floer homology and alternating knots. Geom. Topol., 7:225254, 2003.

[7] P. S. Ozsváth and Z. Szabó. Holomorphic disks and knot invariants. Adv. Math., 186(1):58-116, 2004.

[8] P. S. Ozsváth and Z. Szabó. Holomorphic disks and topological invariants for closed threemanifolds. Ann. of Math. (2), 159(3):1027-1158, 2004.

[9] P. S. Ozsváth and Z. Szabó. Holomorphic triangles and invariants for smooth four-manifolds. Adv. Math., 202(2):326-400, 2006.

[10] P. S. Ozsváth and Z. Szabó. Holomorphic disks, link invariants and the multi-variable Alexander polynomial. Algebr. Geom. Topol., 8(2):615-692, 2008.

[11] J. A. Rasmussen. Floer homology and knot complements. PhD thesis, Harvard University, 2003.

[12] M. Scharlemann and A. Thompson. Heegaard splittings of (surface) $\times$ I are standard. Math. Ann, 295:549-564, 1993.

[13] N. Shirokova. On the classification of Floer-type theories. arXiv:0704.1330.

[14] V. Turaev. Torsions of 3-dimensional manifolds, volume 208 of Progress in Mathematics. Birkhäuser Verlag, Basel, 2002. 
Department of Mathematics, Columbia University, New York 10027

petero@math. columbia.edu

Rényi Institute of Mathematics, Hungarian Academy of Sciences, H-1053 Budapest, REÁltanoda UTCA 13-15, HUNGARY

stipsicz@math-inst.hu

Department of Mathematics, Princeton University, Princeton, New Jersey 08544

szabo@math.princeton.edu 